\title{
Review Article \\ Fractal Reflectarray Antennas: State of Art and New Opportunities
}

\author{
S. Costanzo, F. Venneri, G. Di Massa, A. Borgia, A. Costanzo, and A. Raffo \\ Dipartimento di Ingegneria Informatica, Modellistica, Elettronica e Sistemistica, Università della Calabria, 87036 Rende, Italy \\ Correspondence should be addressed to S. Costanzo; costanzo@deis.unical.it
}

Received 22 July 2016; Accepted 4 September 2016

Academic Editor: Renato Cicchetti

Copyright (C) 2016 S. Costanzo et al. This is an open access article distributed under the Creative Commons Attribution License, which permits unrestricted use, distribution, and reproduction in any medium, provided the original work is properly cited.

\begin{abstract}
Fractal geometries are appealing in all applications where miniaturization capabilities are required, ranging from antennas to frequency selective surfaces (FSS) design. Recently, some fractal patches configurations, giving low losses, reduced size, and quite good phase ranges, have been proposed for the design of reflectarray unit cells. This paper reviews existing fractal-based reflectarrays, highlighting their benefits and limitations. Furthermore, a comprehensive analysis of an innovative reflectarray unit cell, using a fractal-shaped fixed-size patch, is presented. The miniaturization capabilities of the Minkowski fractal shape are fully exploited to obtain a compact cell offering quite good phase agility, by leaving unchanged the patch size and acting only on the fractal scaling factor. Experimental validations are fully discussed on a realized $10 \mathrm{GHz} 0.3 \lambda \times 0.3 \lambda$ cell. This is subsequently adopted to synthesize various reflectarray prototypes offering single or multiple-beam capabilities over a quite large angular region (up to 50 degrees). Finally, experimental validations on a realized $15 \times 15$ elements prototype are presented to demonstrate the wide angle beam-pointing capabilities as well as a quite large bandwidth of about $6 \%$.
\end{abstract}

\section{Introduction}

Reflectarray antennas are low profile reflectors composed of an array of microstrip elements illuminated by a feed antenna [1]. They inherit all appealing features of microstrip technology, such as low cost, easy fabrication, reduced mass, and volume. Furthermore, high efficiencies may be provided thanks to the use of spatial feeding mechanism. Many different reflectarray configurations have been proposed in the literature, each one based on a specific phase tuning of the field reradiated by the single unit cell, such as changing the size, the shape, and/or the position of each radiator [24]. Moreover, the electrical properties of reflectarray elements can be actively tuned, by integrating the unit cells with electrically controllable components, such as varactor diodes or MEMs [5-12]. In these last cases, reflectarrays acquire also reconfiguration capabilities, like beam-scanning and radiation pattern reshaping [10].

Currently, there are several challenges in the design of reflectarray antennas [1], focusing on the operating bandwidth enlargement, losses reduction (especially in the case of active configurations), and unit cell miniaturization, essential for mutual coupling decrease or when requiring wide beamsteering angles. Many passive and active reflectarray configurations have been developed to meet the above demands and most of them are reported and described in [1]

Addressing some of the challenges listed above, fractalbased reflectarray configurations have been recently proposed in the literature [13-19]. Fractal geometries are characterized by the self-similarity property; that is, a certain shape is scaled and repeated a number of times to form the final fractal structure. Practically, a fractal shape is derived by an iterative procedure characterized by a starter shape, that is, the fractal generator, a scaling factor $S$, the dimension of the shape to be scaled, and the iteration number $n$. The application of fractal geometries to conventional antennas allows us to increase their electrical length, thus reducing the overall size of the radiating structure $[20,21]$. These intrinsic miniaturization capabilities of fractal patches are very appealing for the design of reflectarrays and phasedarray antennas. As a matter of fact, the use of a very small array radiator offers many advantages, such as mutual coupling reduction or the capability to work with an array grid characterized by very small interelements spacing. In 
addition, the use of a smaller array lattice greatly increases the bandwidth of reflectarray antennas [22-24], thus ensuring good scanning performances within a larger frequency range.

The fractal geometry concept was already adopted in similar application fields, namely, in frequency selective surfaces (FSS) design [25, 26], offering well established solutions. Just recently, fractals have been applied to reflectarray elements design and so far obtained results can only be considered as preliminary. Despite the fact that all the proposed configurations offer very interesting insights, each of them suffers from some limitations and most of them are not optimized to enhance the bandwidth performances. A Minkowski reflectarray element is proposed in [16] to offer a limited phase tuning range of about $280^{\circ}$, by varying the patch side length from $0.1 \lambda$ to $0.43 \lambda$, where $\lambda$ is the free space wavelength. Other fractal unit cells are presented in the same paper that exploits the multiresonance properties of fractals to give very wide phase ranges, at the expense of a lower miniaturization level and a greater unit cell size. Also in [13], the patch dimension is properly tuned in order to change the unit cell reflection phase. Using these last approaches, the benefits due to the fractal element size reduction are not adequately exploited. As a matter of fact, the unit cell size cannot be properly reduced, since it is fixed by the maximum patch length necessary to realize the desired phase tuning. In order to overcome this last limitation, a fixed-size Minkowski patch is proposed in [17] as reflectarray unit cell. A variable length rectangular slot, sliced in the ground plane, is adopted as phase tuning parameter. This configuration allows a proper miniaturization of the radiating element, with a reflectarray cell offering a phase swing that varies between $313^{\circ}$ and $324^{\circ}$. However, the presence of the slot in the ground plane introduces unwanted back-radiation losses, thus strongly reducing the efficiency of each reflectarray radiator.

An alternative Minkowski fractal reflectarray cell has been recently proposed by the authors in $[18,19]$. As in [17], the patch size is properly fixed during the design stage, while the reflection phase control is realized by varying the scaling factor $S$. This configuration offers a very high miniaturization level, giving the capability to design reduced size reflectarray cells, with sides less than half-wavelength at the working frequency.

In this work, an extensive performances analysis of the proposed configuration is carried out, through the design of different fractal unit cells, having gradually decreasing sizes ranging from $0.6 \lambda \times 0.6 \lambda$ down to $0.3 \lambda \times 0.3 \lambda$, at $10 \mathrm{GHz}$. A significant bandwidth improvement is demonstrated by reducing the unit cell size. Good phase tuning ranges of about $320^{\circ}-345^{\circ}$ are derived in all considered cases. Furthermore, very low losses are obtained with respect to other similar configurations [17]. Finally, the miniaturized $0.3 \lambda \times 0.3 \lambda$ unit cell is adopted to design a set of small reflectarrays, composed by $15 \times 15$ elements illuminated by an offset horn. Each antenna is designed in order to synthesize a radiation pattern with one or multiple-beams directed along very wide direction from broadside. A good agreement is achieved between design constraints and synthesized patterns. Furthermore, successful experimental validations on a reflectarray prototype with a main lobe directed along $50^{\circ}$ in the $H$-plane are presented.

The paper is organized as follows. Section 2 illustrates and discusses the existing fractal reflectarray configurations. Section 3 describes the general design details of an innovative fixed-size fractal-shaped reflectarray element. Section 4 describes the design and the experimental validations of some reduced size fractal-shaped reflectarray prototypes with single and multiple-beam features over quite large angular region. Conclusions are finally outlined in Section 5.

\section{Review on Existing Fractal Reflectarray Elements}

Currently, three general approaches are adopted in the design of fractal reflectarray cells. As usually done in standard reflectarray configurations, the phase tuning of the reradiated field is realized by changing one or more geometrical parameters of the fractal unit cell.

The first approach is based on the use of a variable size fractal patch [13-16], where the reflection phase is tuned by changing the patch dimension around its resonant size. In this case, the miniaturization effect, due to the fractal geometries, allows us to adopt a set of smaller variable size patches to achieve quite good phase variation ranges, with respect to standard variable size square/rectangular configurations printed on the same substrate. However, the patch resizing operation strongly restricts the fractal miniaturization benefits. In the other two approaches, the phase variation mechanisms are independent on the patch size, so offering the opportunity to fully exploit the intrinsic size reduction capabilities of fractals. The use of identical-size smaller radiators, in fact, allows us to reduce mutual coupling level or to implement wider beam-scanning functions. These configurations are based on the use of a fixed-size fractal patch; in the first case [17], this is coupled to a phase tuning element, that is, a variable length slot sliced in the ground plane, which reduces the radiator efficiency, while in the latter case $[18,19]$ the patch is properly reshaped by changing the fractal scaling factor $S$.

Table 1 provides an overview of the main features and benefits offered by the fractal approaches summarized above. Each technique is described with more details in the following sections.

2.1. Variable Size Reflectarray Fractal Elements. The first numerical investigation on variable size fractal reflectarray patches has been performed in [13], where a few 1st iteration fractal elements, respectively, based on Koch-triangle, Kochsquare, and Minkowski shapes (Figure 1(a)), are proposed and analyzed. The analysis shows that all considered configurations provide very low losses, reaching their maximum value of $-0.35 \mathrm{~dB}$ in the case of the Minkowski patch (Figure 1(c)). Furthermore, the fractal cells offer relative small phase ranges, with variations from $250^{\circ}$ up to $300^{\circ}$ (Figure 1(b)). In particular, the Minkowski radiator provides the largest phase range, against a $\pm 20 \%$ patch size variation around resonance, at the expense of a greater but acceptable sensitivity to fabrication errors (Figure 1(b)). As claimed by the authors, the reduced phase ranges, offered by the 
TABLE 1: Selected approaches for fractal reflectarray unit cell implementation and qualitative analysis of a few related features.

\begin{tabular}{|c|c|c|c|}
\hline Phase tuning approach & Miniaturization level & Phase range & Reflection loss \\
\hline \multicolumn{4}{|l|}{ Variable size fractal patch } \\
\hline Single layer & Moderate & Moderate & Low \\
\hline Dual-layer or multiband & Poor & Very good & Low \\
\hline Fixed size fractal patch with variable length slot & Good & Good & High \\
\hline Fixed size fractal patch with variable scaling factor & Very good & Good & Low \\
\hline
\end{tabular}

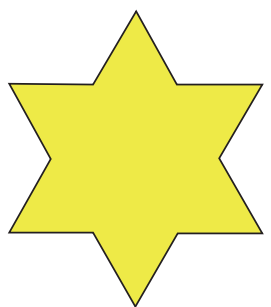

Koch-triangle

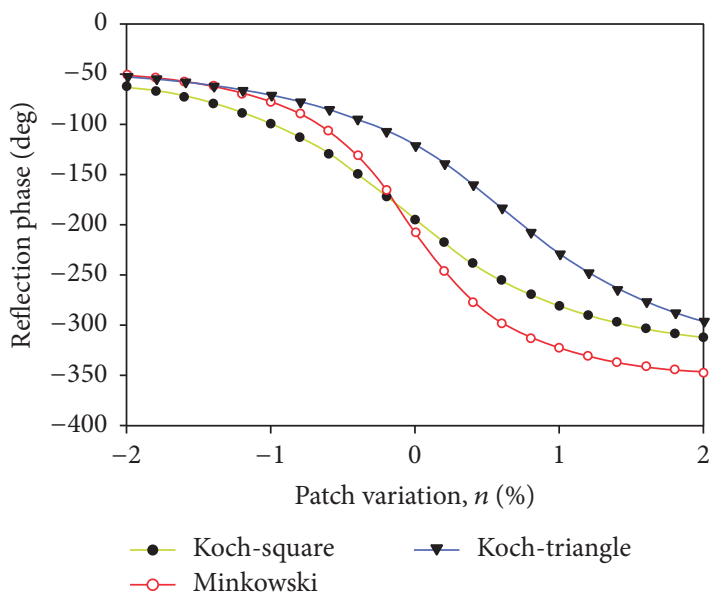

(b)

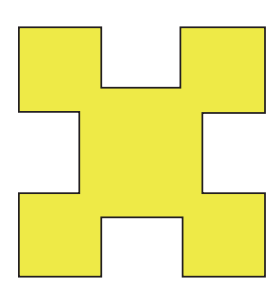

Minkowski

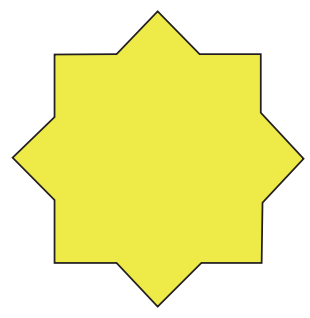

Koch-square

(a)

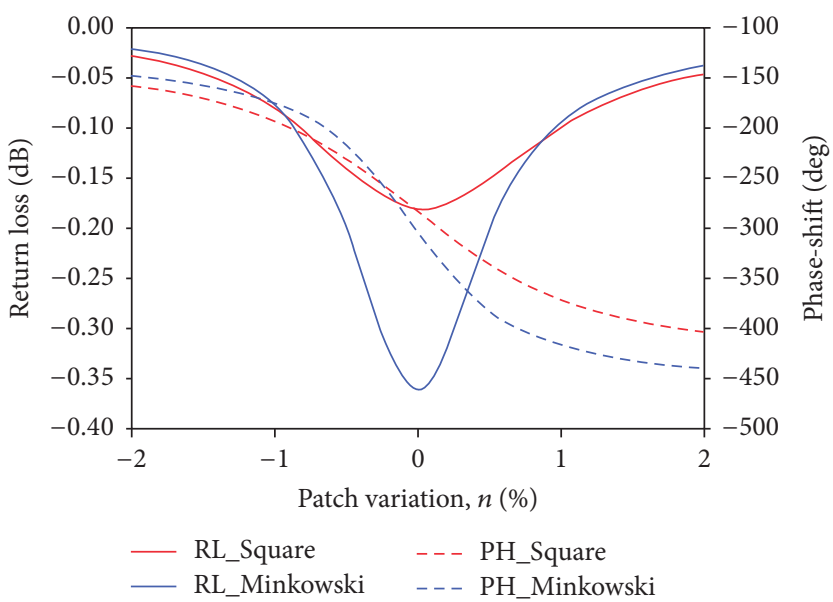

(c)

FIGURE 1: Simulated fractal reflectarray unit cells $\left(f_{0}=11 \mathrm{GHz}, \varepsilon_{r}=3.54\right.$, and thickness $\left.=1.524 \mathrm{~mm}\right)$ : (a) variable size fractal patches; (b) comparison between reflection phase curves versus patch variation percentage ( $n \%)$ computed for Koch-square, Koch-triangle, and Minkowski patches; (c) comparison between reflection coefficient responses versus $n \%$ computed for Minkowski and square patches.

configurations depicted in Figure 1, could not be sufficient for a practical reflectarray design, and they can be partially increased by extending the patch variation percentage.

A fractal-shaped configuration is able to reduce the dimensions of patches giving the required reflection phase range (Figure 1(c)) [14], if compared to standard square/rectangular radiator; however, a patch variation percentage greater than $\pm 20 \%$ may cancel some of the benefits due to fractals miniaturization capabilities, namely, the mutual coupling reduction or the opportunity to work with unit cell sizes less or equal to half-wavelength (useful for wide beam-scanning implementation). Furthermore, the use of reflectarray radiators having very different dimensions from each other may affect the accuracy of the infinite array analysis approach in the mutual coupling estimation.
An alternative way to increase the phase range of variable size fractal reflectarray configurations is suggested in [15], where a dual layer Minkowski cell is designed, offering a smooth reflection phase curve over a range of about $450^{\circ}$. However, this last structure is a little more complicated with respect to a single layer cell, and its demonstration is actually limited to the numerical domain.

Further examples of variable size fractal reflectarray cells are reported in [16]. The aim of this last study is to achieve a phase range larger than $360^{\circ}$, together with a smaller slope in the reflection phase curve as a function of the element size. Starting from the purpose to increase the small phase range $\left(\cong 280^{\circ}\right)$ achieved with a $10 \mathrm{GHz}$ Koch island fractal (equivalent to a Minkowski patch with a square indentation, see [16]), the authors propose two innovative multiresonance 

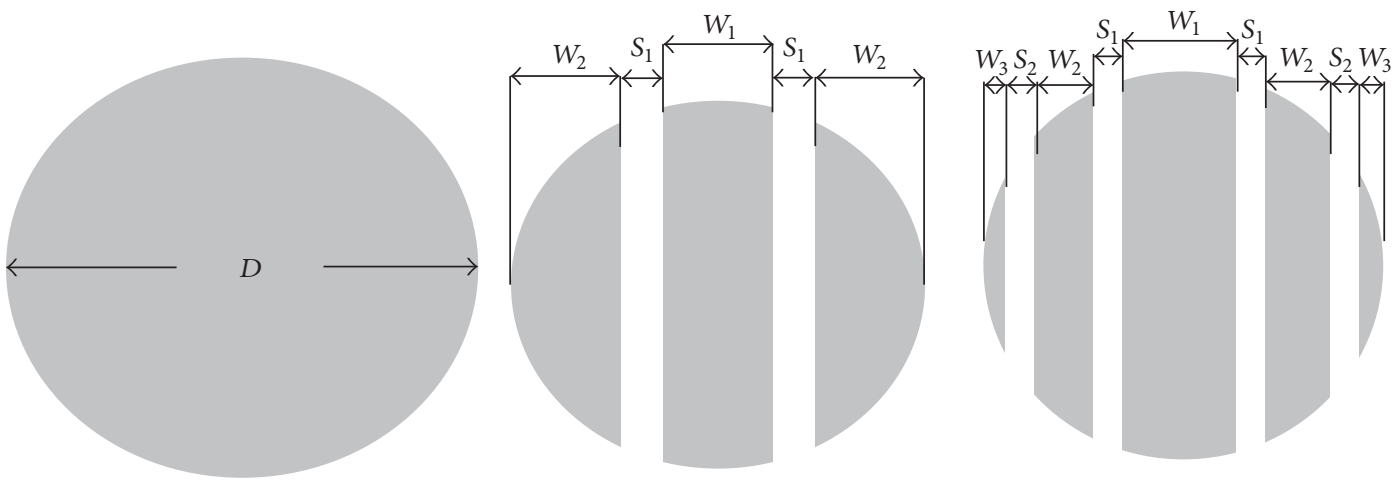

(a)

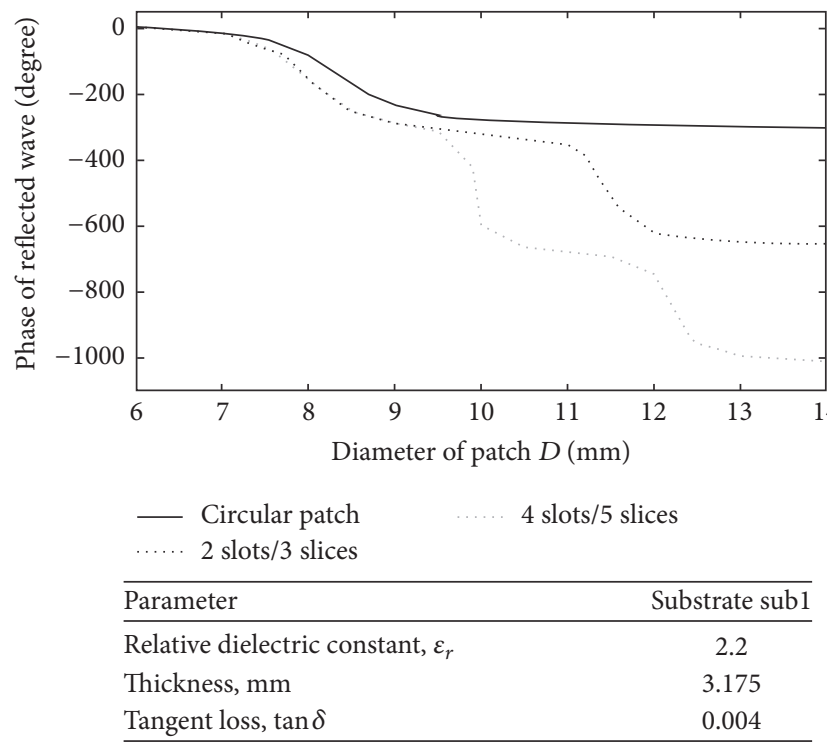

(b)

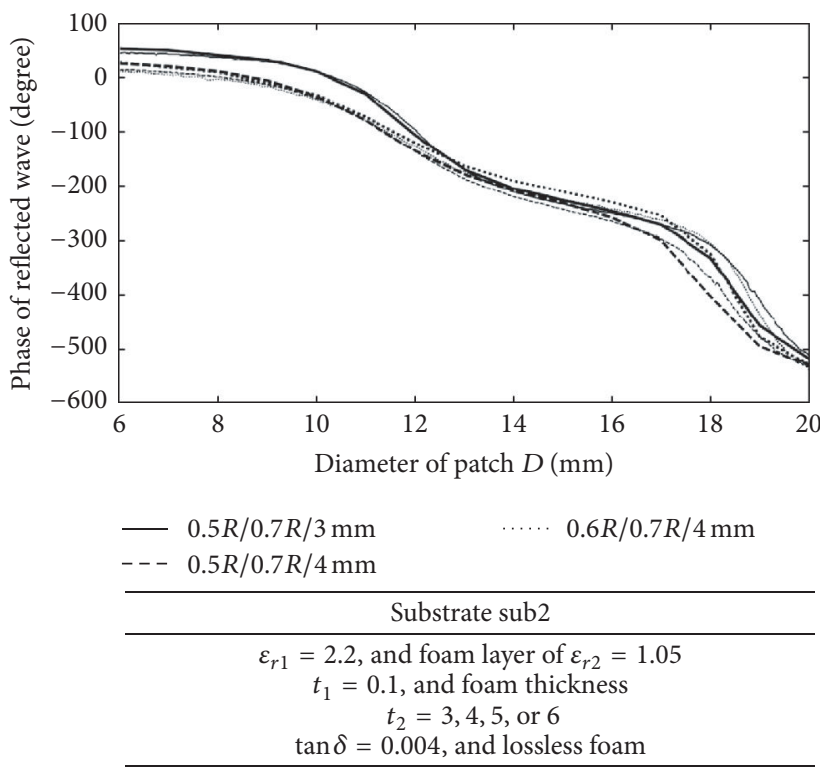

(c)

FiguRE 2: (a) Sliced-circular fractal; (b) simulated phase response versus patch diameter of circular patch and sliced-circular fractals with two-slots and four-slots $\left(f_{0}=10 \mathrm{GHz}\right)$; (c) linearized phase responses of two-slot sliced-circular fractal for different slices widths and foam substrate layer thickness.

fractal elements. The first solution consists of a circular patch divided into three slices, by introducing two vertical slots symmetrically located with respect to the patch centre (Figure 2(a)), while the second configuration is obtained by applying the same concept of slicing to a square microstrip patch to yield the fractal structures shown in Figure 3(a). In this last case, the lengths $L_{i}$, widths $W_{i}$, and separations $S_{i}$ of the slices can be varied independently, while keeping the structure within the $L \times L$ square [16].

Both the structures provide a wider phase tuning range at $10 \mathrm{GHz}$ (Figures 2(b) and 3(b)), due to the multiband behavior of the sliced patches. However, the very high slopes characterizing the curves hardly reduce the bandwidth and the fabrication tolerances of the cells, so requiring further optimizations whose primary target is the phase response linearization. To this end, different parametric analyses are performed, involving cell size, slots positions and widths, substrate thickness, and stratification. The results returned by the analysis are satisfactory in terms of phase range extension and curve slopes (Figures 2(c) and 3(c)), at the expense of a too large patch size variation and, consequently, a greater cell size (i.e., $0.7 \lambda \times 0.7 \lambda$ at $10 \mathrm{GHz}$ ), so inhibiting the possibility to work with a smaller array lattice. Furthermore, as illustrated in Figures 2(c) and 3(c), the required element size variation corresponds to a ratio, greatest/smallest patch, ranging from 3.3 up to 5, that will affect the infinite array approach validity and the accuracy in the unit cell characterization, especially in relation to the mutual coupling estimation.

2.2. Fixed-Size Reflectarray Fractal Elements. A different class of miniaturized reflectarray unit cells is introduced in [17], where a fixed-size Minkowski element is proposed. The phase swing is achieved through a variable length slot, sliced in the ground plane at the centre of the patch (Figure 4(a)). This approach benefits from the fixed size of the radiator that allows us to better exploit the miniaturization ability of fractal 

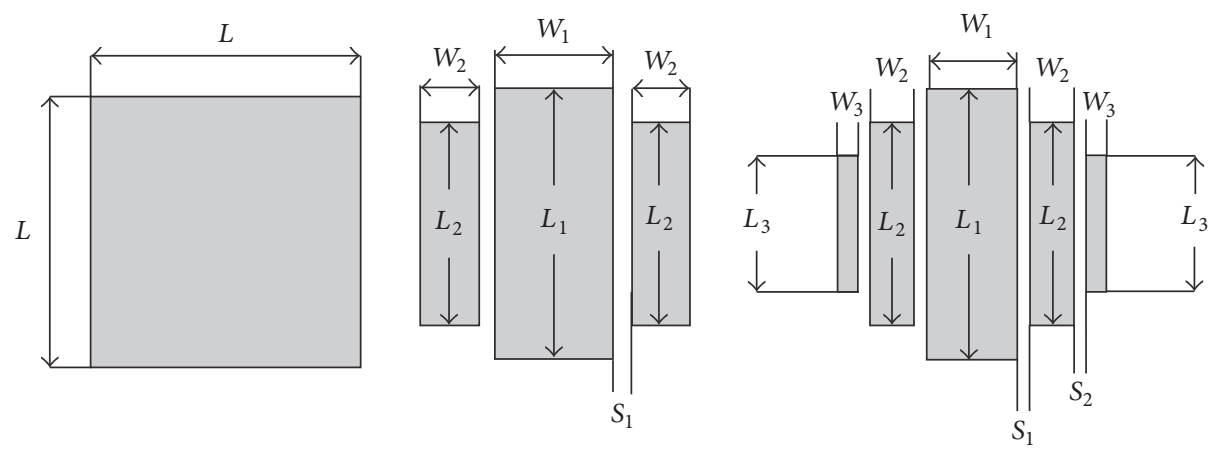

(a)

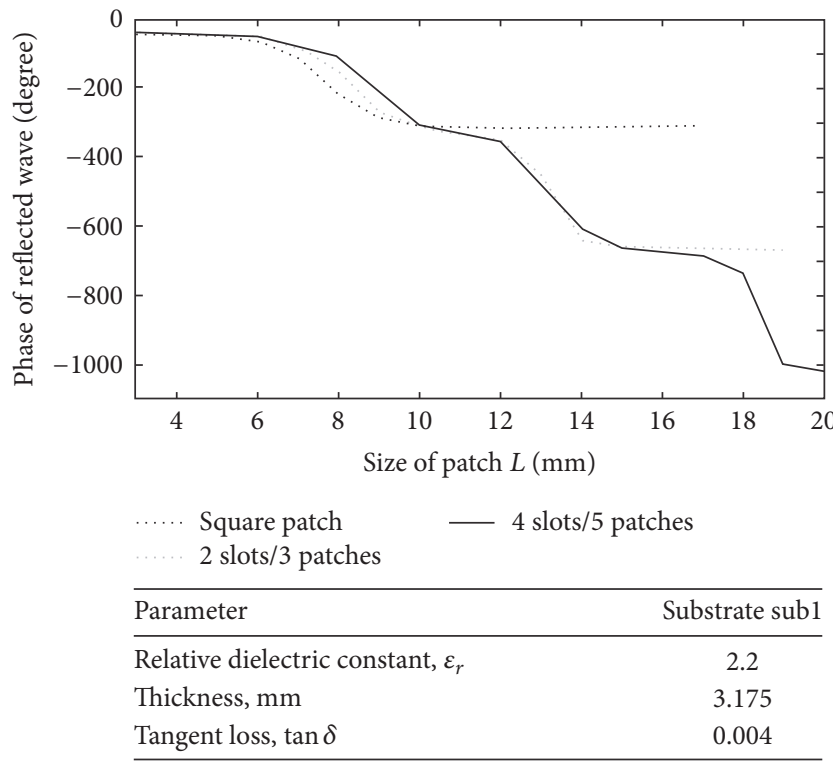

(b)

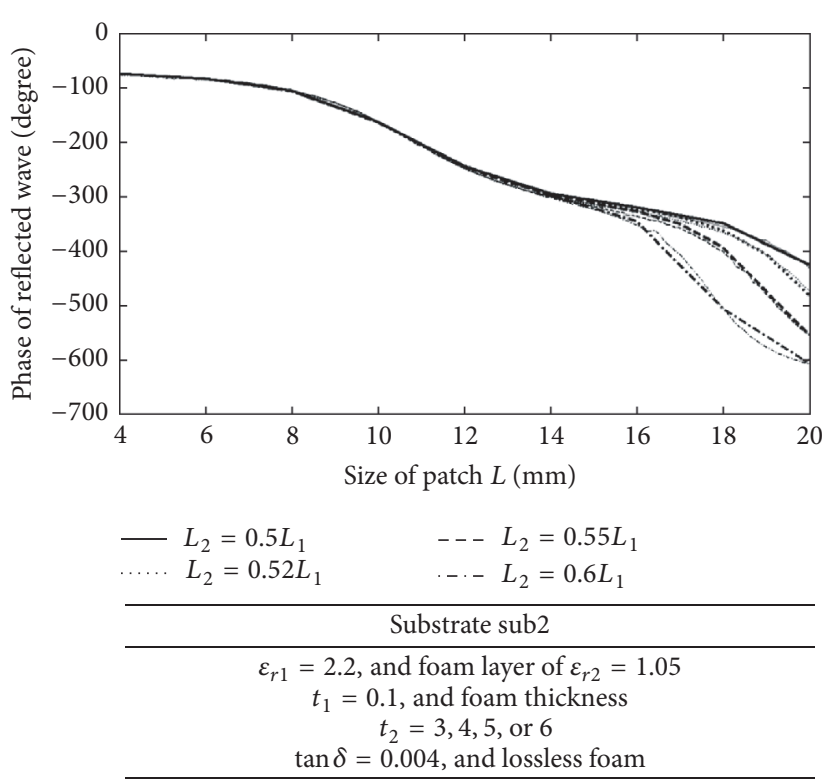

(c)

FIGURE 3: (a) Multislice fractal square patch; (b) simulated phase response versus patch size of square patch and multislice fractals with twoslots and four-slots $\left(f_{0}=10 \mathrm{GHz}\right)$; (c) linearized phase responses of two-slots multislice fractal for different patches lengths (foam substrate layer thickness $=5 \mathrm{~mm})$.

patches. Both the 1st- and the 2nd-order Minkowski fractal patch-slots are considered. Patch dimensions at $11.3 \mathrm{GHz}$ are reported in Figure 4, while the slot length $L_{s}$ is varied from $0 \mathrm{~mm}$ up to $6 \mathrm{~mm}$. As $L_{s}$ increases, the resonant frequency of the cell decreases and the maximum reflection loss rises. The effects of the slot length variation on the unit cell response are illustrated in Figure 5, where the simulated reflection coefficients for square, 1st and 2nd Minkowski patches, are reported, respectively. The simulated results depicted in Figure 5 are experimentally validated in [17].

The above fractal-shaped unit cells allow us to reduce the resonant frequency, which is equivalent to a miniaturization effect. Furthermore, it can be observed that the fractal shapes increase the maximum phase swing at the expense of higher curve slopes, thus reducing the cell bandwidth. The 1st iteration cell reaches $313^{\circ}$ and the 2 nd iteration one offers a phase range of about $324^{\circ}$. However, the slot in the ground plane introduces unwanted back-radiations that increase reflection losses up to $3.3 \mathrm{~dB}$ and $4.1 \mathrm{~dB}$ for the 1 st and the 2 nd iteration cells, respectively.

In order to overcome the above drawback related to the fractal patch-slot configuration and to take advantage from the fixed-size approach, an alternative Minkowskibased configuration is proposed in $[18,19]$. The patch size is properly fixed to achieve the desired miniaturization level, while the phase variation mechanism is implemented by varying the fractal scaling factor $S$. This configuration, as the previous one, allows us to fully exploit the miniaturization effect of fractal geometries. Furthermore, as demonstrated in [19], it can offer, at the same time, very small size radiators, good phase swings, and lower reflection losses. By reducing the unit cell size, that is, the array interelement spacing, the reflection phase curves slope decreases, so improving also the bandwidth performances. Overall details on this configuration are reported in the next paragraph, where an extended analysis is performed and discussed in order to 


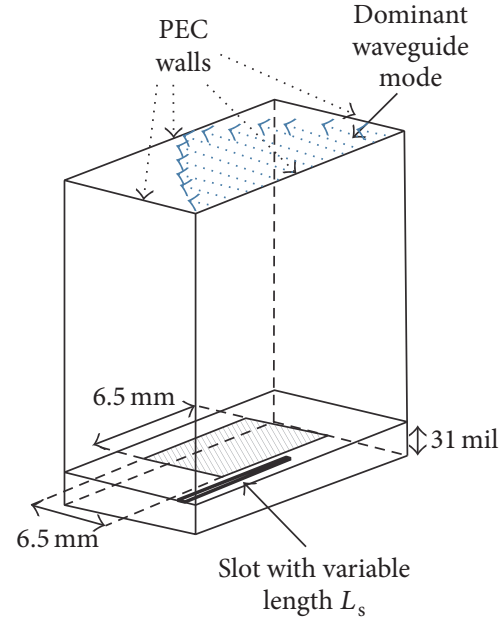

(a)

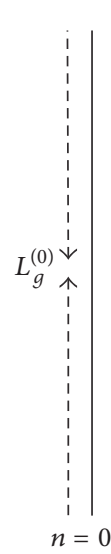

$=0$

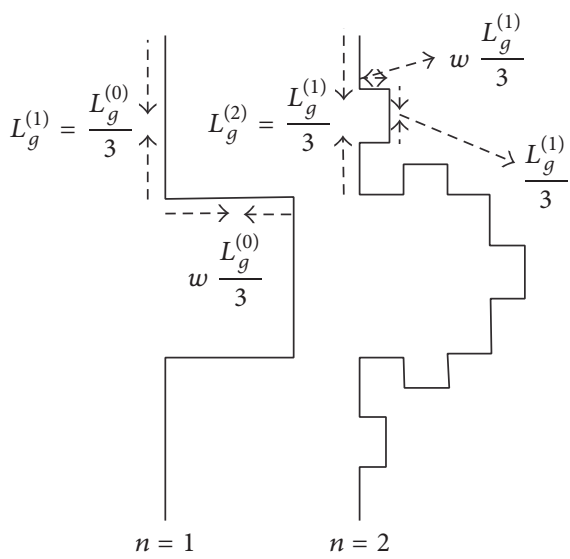

(b)

FIgURE 4: (a) Simulated reflectarray patch-slot unit cell; (b) generation procedure for the Minkowski fractal curves with $n=0,1,2$ corresponding to the generator, 1st and 2nd Minkowski configurations.

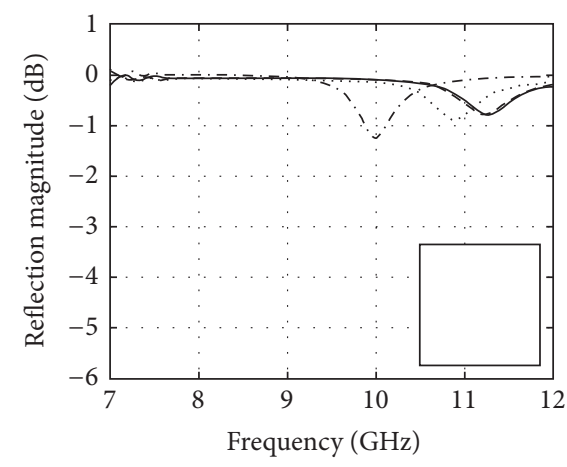

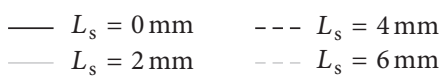

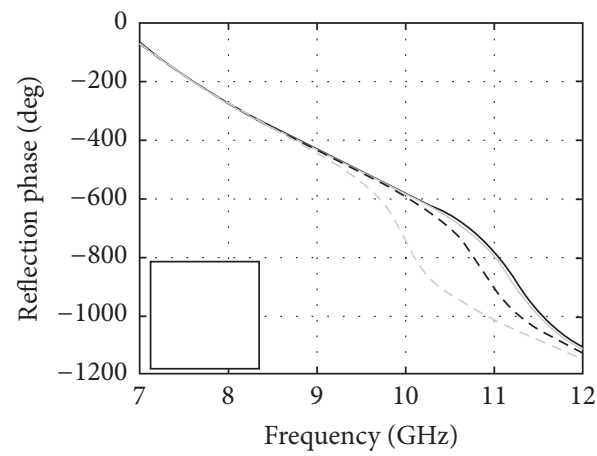

$\begin{aligned}-L_{\mathrm{s}} & =0 \mathrm{~mm} \quad--L_{\mathrm{s}}=4 \mathrm{~mm} \\ L_{\mathrm{s}} & =2 \mathrm{~mm}\end{aligned}$

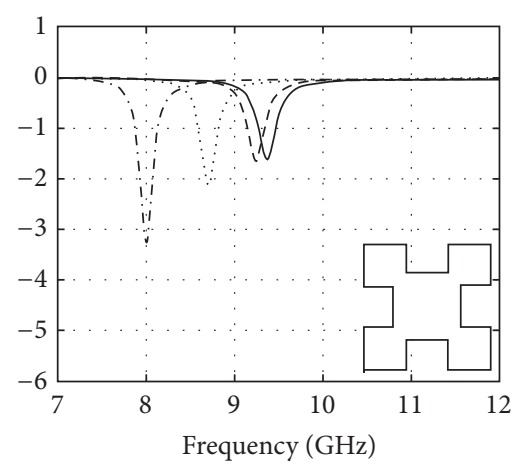

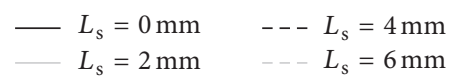

(a)

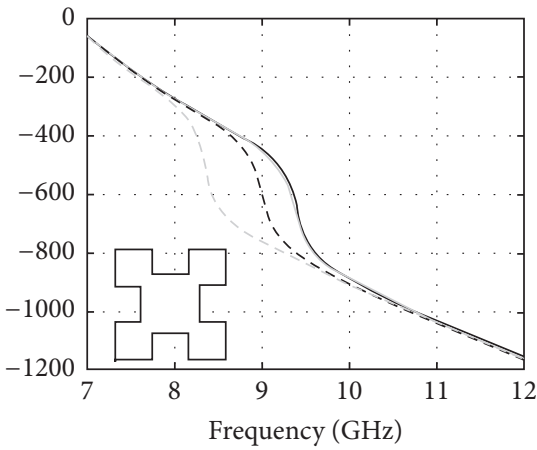

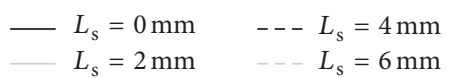

(b)
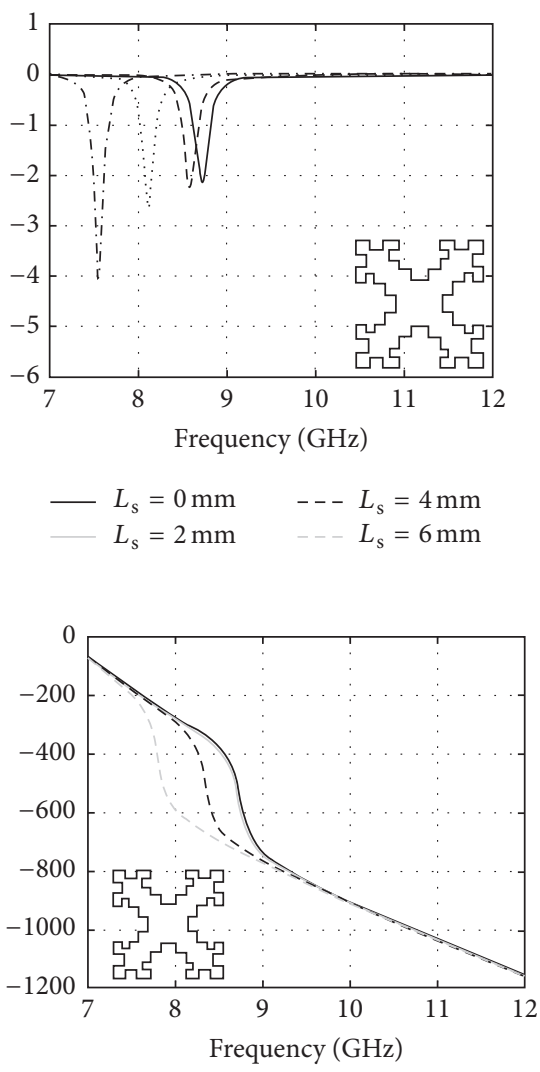

$\begin{aligned}-L_{\mathrm{s}} & =0 \mathrm{~mm} \quad--L_{\mathrm{s}}=4 \mathrm{~mm} \\ L_{\mathrm{s}} & =2 \mathrm{~mm}\end{aligned}$

FIGURE 5: Simulated reflection magnitude (a) and phase (b) for a square, 1st and 2nd Minkowski patches for different slot lengths. 


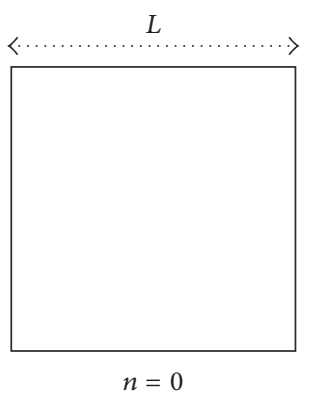

(a)

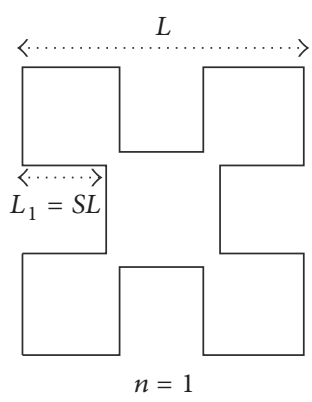

(b)

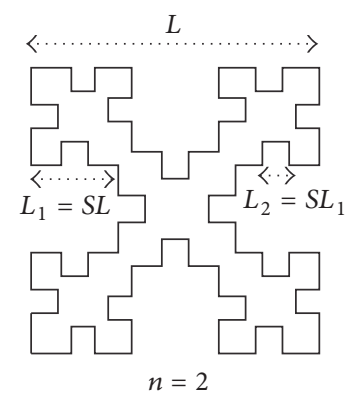

(c)

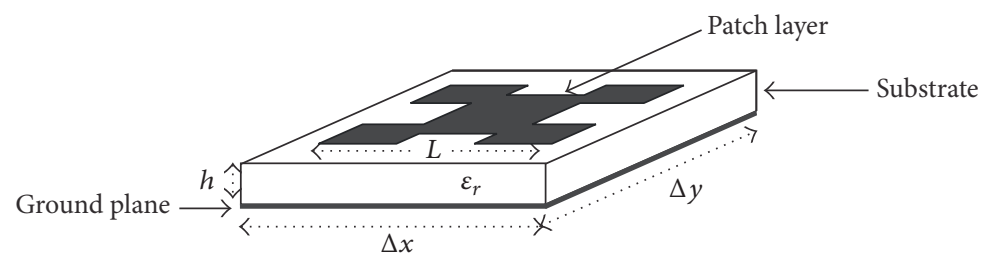

(d)

Figure 6: Minkowski fractal geometry: (a) generator square patch; (b) first iteration patch; (c) second iteration patch; and (d) unit cell layers.

highlight the benefits related to the proposed unit cell, in terms of miniaturization capabilities, reflection loss, phase range, bandwidth, and, finally, practical design of wide beamscanned or multibeam reflectarrays.

\section{Fixed-Size Fractal Element with Variable Scaling Factor}

The reflectarray unit cell, preliminary proposed by the authors in $[18,19]$, is based on the use of the Minkowski fractal patch illustrated in Figure 6. The adopted fractal shape is characterized by a beginning square element of dimensions $L \times L$ (Figure 6(a)), which can be iteratively modified according to the following construction rule. In the first iteration $(n=1)$, a smaller square of side $S L$ is removed from the centre of each side of the initial square patch. $S$ is called the scaling factor, variable in the range from 0 up to $L / 3$. The structure resulting from the first iteration is illustrated in Figure 6(b). By applying the described construction rule to each side of the above structure, the 2nd iteration patch is obtained (Figure 6(c)). The fractal construction can be infinitely reiterated to obtain an increasingly complex self-similar shape.

As demonstrated by the contributions described in the previous paragraph, the main benefit derived from the adoption of fractal geometries in the design of microstrip antennas is that more electrical length can be fitted into a smaller physical area $[20,21]$. Of course, the increased electrical length of the fractal patch leads to a lower resonant frequency; then the fractal antenna must be miniaturized in order to obtain the resonance at the desired working frequency. In the case of the Minkowski fractal patch (Figures 6(b) and 6(c)), for example, the resonant side length is longer with respect to the square patch with the same straight length $L$ (Figure 6(a)). As a matter of fact, the fractal patch length is given by the following equation [20]:

$$
L_{n}=(1+2 S)^{n} L,
$$

where $n$ is the iteration number and $L_{n}$ is the resonant length of the patch generated at $n$th iteration. It can be observed that, for a fixed value of $n$, the length $L_{n}$ increases when a greater scaling factor $S$ is considered. Furthermore, the length of Minkowski patches increases when the fractal construction is progressively reiterated, namely, for greater values of $n$.

Keeping in mind the above fractal geometries features and remembering that one of the most adopted phase tuning technique for reflectarrays design is based on the use of variable size square/rectangular patches, the Minkowski patch is proposed in [18] as reflectarray radiator able to control its phase response simply by varying its resonant length. However, unlike square/rectangular patches, the electrical length of the fractal resonator can be tuned without altering its straight length $L$ (Figure 6), namely, without changing the area covered by the antenna.

As a matter of fact, the unit cell phase tuning proposed in [18] is realized by varying the scaling factor $S$, while the patch size $L$ is properly fixed in order to match the resonance condition at the desired operating frequency in correspondence of a prescribed value of $S$, which gives the desired miniaturization level. Therefore, the proposed approach allows us to work with reflectarray elements characterized by the same side length. In this way, the miniaturization effects due to the fractal geometry can be properly exploited in order to reduce the reflectarray interelement spacing, thus allowing us to maximize the scan angle in those applications requiring beam-scanning capabilities [21] or to synthesize a multibeam pattern able to concentrate the radiated power along very far directions from each other.

3.1. Design of 1st Iteration Fractal Unit Cells. The proposed phase tuning approach is adopted in [19] to design a $10 \mathrm{GHz}$ reflectarray unit cell based on the use of a 1st iteration Minkowski patch (Figure 6(b)). The antenna is printed on a Diclad 870 substrate with a dielectric constant $\varepsilon_{r}=2.33$ and 


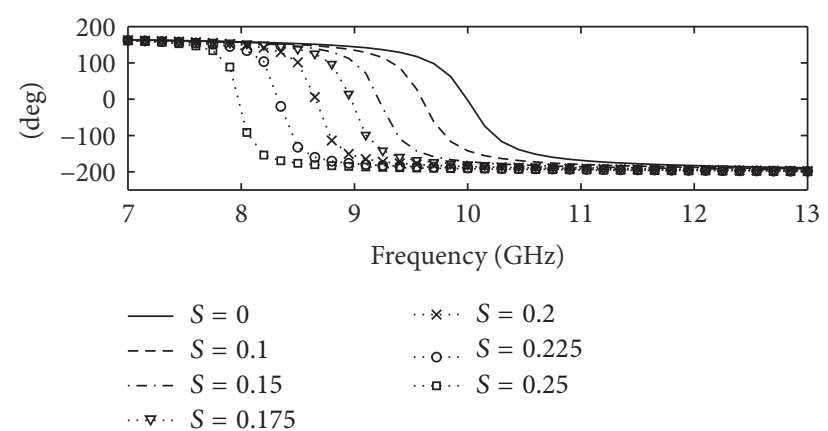

(a)

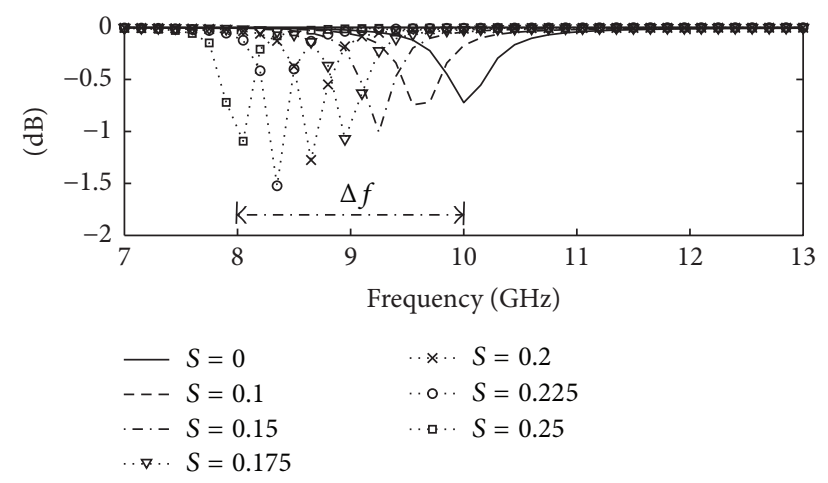

(b)

Figure 7: (a) Phase and (b) amplitude of the simulated reflection coefficient versus frequency for different value of the scaling factor $S$ (1st iteration fractal unit cell with $L=9.215 \mathrm{~mm}$ and $\Delta x \times \Delta y=$ $\left.0.6 \lambda_{0} \times 0.6 \lambda_{0}\right)$.

a thickness $h=0.762 \mathrm{~mm}$ (Figure 6(d)). A commercial fullwave code, based on the infinite array approach, is adopted as design tool. A normally incident plane wave is fixed as source. The unit cell size is initially set to a value of $\Delta x \times \Delta y=$ $0.6 \lambda_{0} \times 0.6 \lambda_{0}$ at the design frequency $f_{0}$ (Figure $6(\mathrm{~d})$ ).

A $10 \mathrm{GHz}$ resonant square patch $(L=9.215 \mathrm{~mm})$ is considered as generator for the fractal unit cell design (Figure 6(a)). For the simplicity of reading, some results already illustrated in $[18,19]$ are reported in the following discussion.

Figure 7 shows the simulated reflection coefficient of the square patch, which corresponds to the curves labeled with $S=0$. In the same figure, the reflection coefficients computed for the 1st iteration fractal patch (Figure 6(b)) and obtained by removing a smaller square of side $S L$ from each side of the generator patch are reported. It can be observed that, by increasing the scaling factor $S$ from 0 up to 0.25 , the resonant frequency of the antenna is progressively reduced from the initial design frequency $f_{0}=10 \mathrm{GHz}$ down to a value of about $8 \mathrm{GHz}$.

As illustrated by (1), this behavior is due to the increased resonant length of the fractal patch, resulting from the application of a greater scaling factor. This frequency swift $\Delta f$ (Figure $7(b)$ ) can be properly exploited to realize a complete phase tuning of the reflectarray unit cell. As a matter of fact, Figure 7 (a) shows that, by varying the value of $S$ from 0 up to

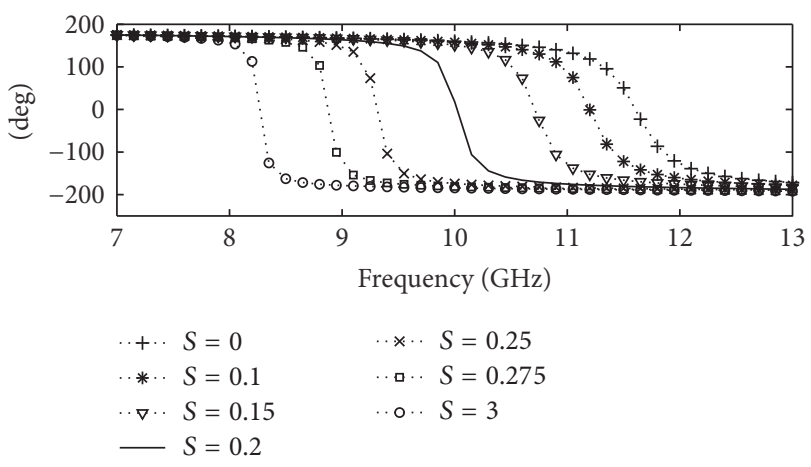

(a)

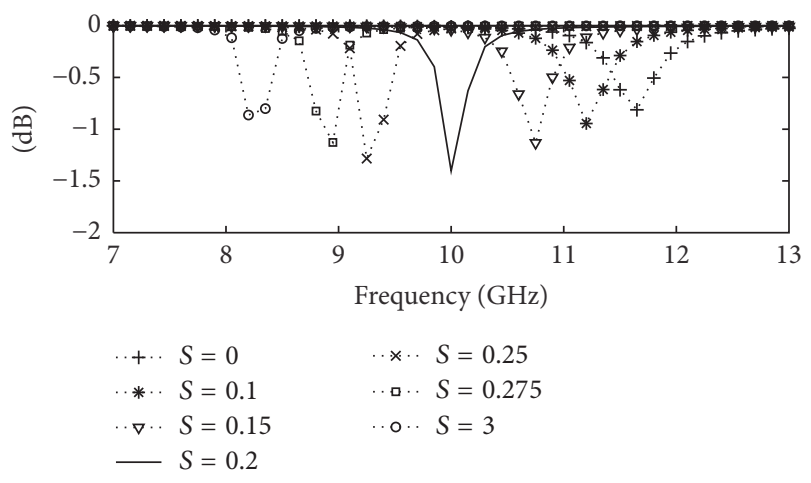

(b)

Figure 8: (a) Phase and (b) amplitude of the simulated reflection coefficient versus frequency for different value of the scaling factor $S$ (1st iteration fractal unit cell with $L=7.86 \mathrm{~mm}$ and $\Delta x \times \Delta y=$ $\left.0.6 \lambda_{0} \times 0.6 \lambda_{0}\right)$.

0.25 , the reflection phase can be tuned within a range of about $360^{\circ}$ at those frequencies lying in a neighborhood of $9 \mathrm{GHz}$.

In order to design a reflectarray unit cell with a maximum phase range at the operating frequency $f_{0}$, the above fractal element is properly resized. To this end, the patch length $L$ is set to a smaller value equal to $7.86 \mathrm{~mm}$, which gives the resonance at $10 \mathrm{GHz}$ for $S=0.2$. Figure 8 shows the reflection coefficient relative to this last fractal element, for different values of the scaling factor $S$. As expected, in the new case the frequency swing is centred around $f_{0}$, so giving a maximum phase range of about $350^{\circ}$ in the neighborhood of $10 \mathrm{GHz}$ (Figure 8(a)). As a consequence of this, it can be affirmed that the adopted configuration allows us to obtain a reflectarray element with a reduced size and a quite good phase tuning range, which is very near to the expected value of $360^{\circ}$. Furthermore, the designed unit cell exhibits a maximum loss of about $1.4 \mathrm{~dB}$ (Figure $8(\mathrm{~b})$ ), which is lower as compared to the maximum loss values achieved with the fractal patch-slots configuration proposed in [17].

Figure 9 shows the unit cell design curve $R(S)$, computed by varying $S$ from 0 up to 0.325 at the operating frequency $f_{0}=10 \mathrm{GHz}$. A full variation of the reflection phase can be observed, which is a necessary requirement for the design of high performances reflectarray antennas. 


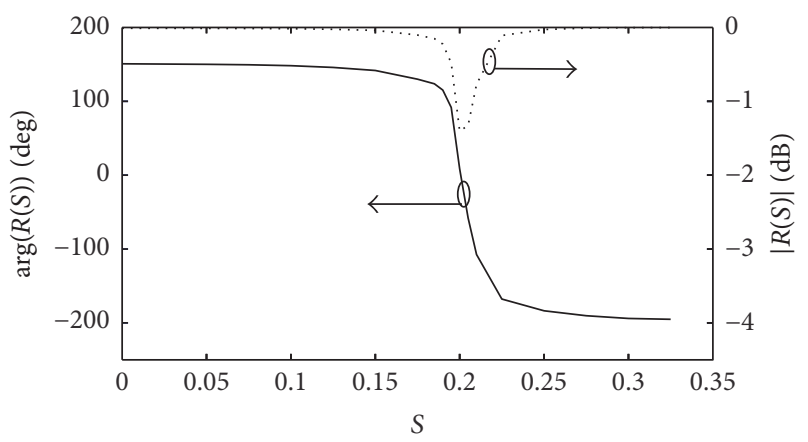

Amplitude

- Phase

FIGURE 9: Simulated reflection coefficient versus $S(L=7.86 \mathrm{~mm}$, $\Delta x \times \Delta y=0.6 \lambda_{0} \times 0.6 \lambda_{0}$, and $f_{0}=10 \mathrm{GHz}$ ).

3.2. Design of 1st Iteration Fractal Unit Cells with Reduced Size. The proposed phase tuning mechanism, based on the variation of the scaling factor $S$, allows us to exploit the miniaturization effect of the Minkowski fractal geometry. As a matter of fact, the intrinsic smaller resonant length of the fractal patch allows us to reduce the unit cell size (i.e., $\Delta x \times \Delta y$, see Figure 6), so giving the capability to design reflectarray antennas with very wide scan angles, as well as larger bandwidth performances [22-24].

In order to investigate the effects due to the cell size reduction, the proposed fractal configuration is further analyzed, by considering the following unit cell sizes: $0.5 \lambda_{0} \times 0.5 \lambda_{0}$, $0.4 \lambda_{0} \times 0.4 \lambda_{0}$, and $0.3 \lambda_{0} \times 0.3 \lambda_{0}$. In the last two cases, the cell dimension affects the resonance of the embedded radiating element. This fact is due to the mutual coupling between the elements that increases when the cell size is reduced. In order to reset the resonance frequency $f_{0}$ to the desired $10 \mathrm{GHz}$ value, a little tuning of the fractal patch length $L$ is performed, obtaining the following new values: $L=7.82 \mathrm{~mm}\left(0.4 \lambda_{0}\right.$ cell $)$, $L=7.6 \mathrm{~mm}\left(0.3 \lambda_{0}\right.$ cell $)$. Conversely, the patch length remains equal to $7.86 \mathrm{~mm}$ for the $0.5 \lambda_{0}$ cell.

The simulated reflection coefficients of the considered structures are reported in Figures 10, 12, and 14, respectively. The aforementioned figures illustrate the reflection coefficient behavior versus frequency, for different values of the scaling factor $S$. Furthermore, Figures 11, 13, and 15 depict the design curves computed by varying $S$ at the central frequency $f_{0}=$ $10 \mathrm{GHz}$. In all cases, a little reduction of the phase tuning range can be observed, with respect to the one obtained when the element is embedded into the $0.6 \lambda_{0}$ cell (Figure 9). In particular, the maximum phase range is equal to about $345^{\circ}$, $340^{\circ}$, and $320^{\circ}$, respectively, for the cell size $0.5 \lambda_{0} \times 0.5 \lambda_{0}$ (Figure 11), $0.4 \lambda_{0} \times 0.4 \lambda_{0}$ (Figure 13), and $0.3 \lambda_{0} \times 0.3 \lambda_{0}$ (Figure 15). However, these phase tuning ranges are acceptable for most applications and they can be considered quite good with respect to the values obtained in other works proposing the use of fractal reflectarray configurations $[13,14,16]$.

Furthermore, a significant reduction of the maximum reflection losses, at $f_{0}=10 \mathrm{GHz}$, can be appreciated in Figures $10(\mathrm{~b}), 12(\mathrm{~b})$, and $14(\mathrm{~b})$ that are equal to $1 \mathrm{~dB}, 0.7 \mathrm{~dB}$, and $0.4 \mathrm{~dB}$, for the $0.5 \lambda_{0}, 0.4 \lambda_{0}$, and $0.3 \lambda_{0}$ cells, respectively.

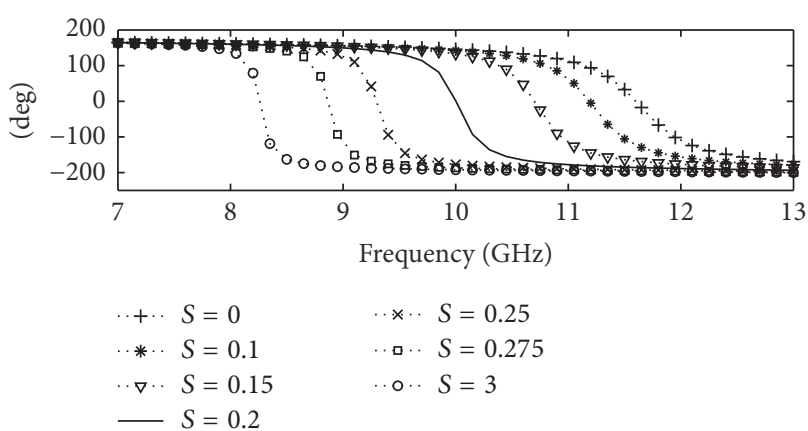

(a)

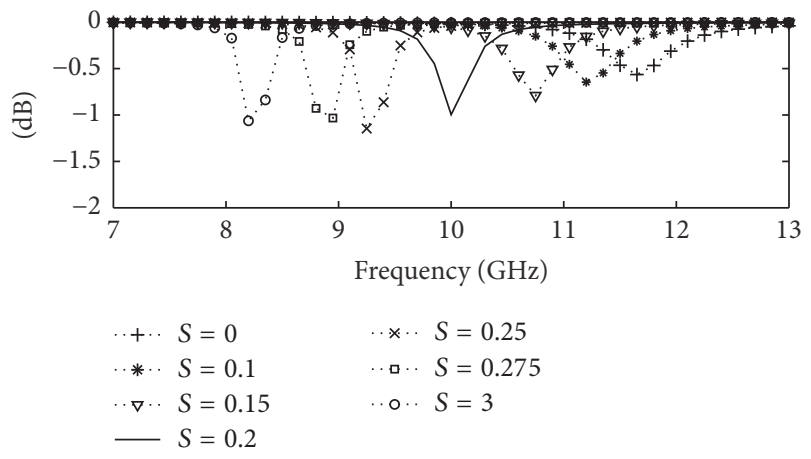

(b)

FIgURE 10: (a) Phase and (b) amplitude of the simulated reflection coefficient versus frequency for different value of the scaling factor $S$ (1st iteration fractal unit cell with $L=7.86 \mathrm{~mm}$ and $\Delta x \times \Delta y=$ $\left.0.5 \lambda_{0} \times 0.5 \lambda_{0}\right)$.

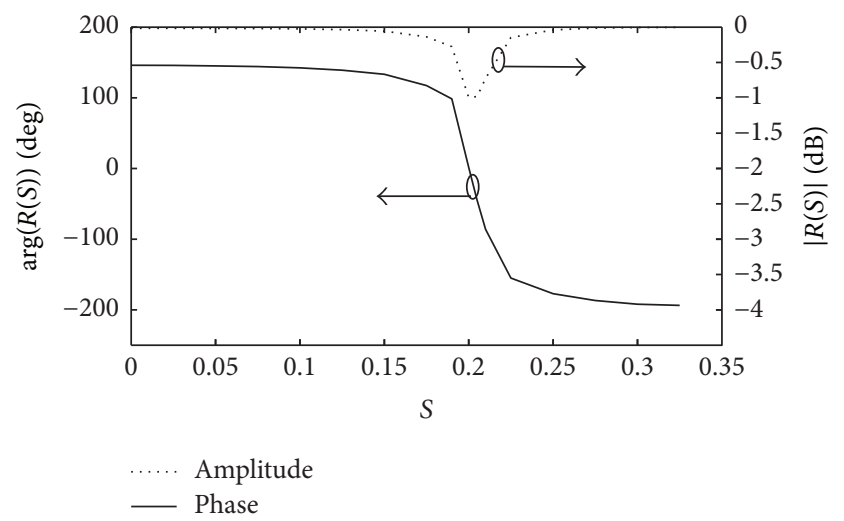

FIGURE 11: Simulated reflection coefficient versus $S(L=7.86 \mathrm{~mm}$, $\Delta x \times \Delta y=0.5 \lambda_{0} \times 0.5 \lambda_{0}$, and $f_{0}=10 \mathrm{GHz}$ ).

The patch size, phase range length, and maximum losses of the three designed unit cells are summarized in Table 2.

In the same table, the cells are compared with two variable size $10 \mathrm{GHz}$ square patches, respectively, embedded in two standard-size $0.6 \lambda_{0} \times 0.6 \lambda_{0}$ and $0.5 \lambda_{0} \times 0.5 \lambda_{0}$ cells. In these last cases, the phase tuning is achieved through the patches size sweep of $\pm 20 \%$ of their resonance length.

The elements size reduction, due to fractal geometry, is computed with respect to the resonating square element obtained for the $0.6 \lambda_{0}$ cell (i.e., $L=9.215 \mathrm{~mm}$ ). A quite 
TABLE 2: Fractal unit cell performances (1st iteration Minkowski patch).

\begin{tabular}{|c|c|c|c|c|c|}
\hline Element Configuration & $\begin{array}{l}\text { Cell size } \\
\Delta x \times \Delta y\end{array}$ & $\begin{array}{c}\text { Resonant } \\
\text { patch length } \\
{[\mathrm{mm}]}\end{array}$ & $\begin{array}{c}\text { Phase range } \\
\text { [deg] }\end{array}$ & $\begin{array}{l}\text { Max loss } \\
{[\mathrm{dB}]}\end{array}$ & $\begin{array}{l}\text { Fractal size reduction } \\
\text { (with respect to the } 0.6 \lambda \\
\text { resonant square patch) }\end{array}$ \\
\hline Variable size square patch & $0.6 \lambda \times 0.6 \lambda$ & 9.215 & 330 & 0.7 & - \\
\hline Variable size square patch & $0.5 \lambda \times 0.5 \lambda$ & 9.15 & 317 & 0.5 & - \\
\hline 1st iteration fractal patch & $0.6 \lambda \times 0.6 \lambda$ & 7.86 & 350 & 1.4 & $14.70 \%$ \\
\hline 1st iteration fractal patch & $0.5 \lambda \times 0.5 \lambda$ & 7.86 & 345 & 1 & $14.70 \%$ \\
\hline 1st iteration fractal patch & $0.4 \lambda \times 0.4 \lambda$ & 7.82 & 340 & 0.7 & $15.14 \%$ \\
\hline 1st iteration fractal patch & $0.3 \lambda \times 0.3 \lambda$ & 7.6 & 320 & 0.4 & $17.53 \%$ \\
\hline
\end{tabular}

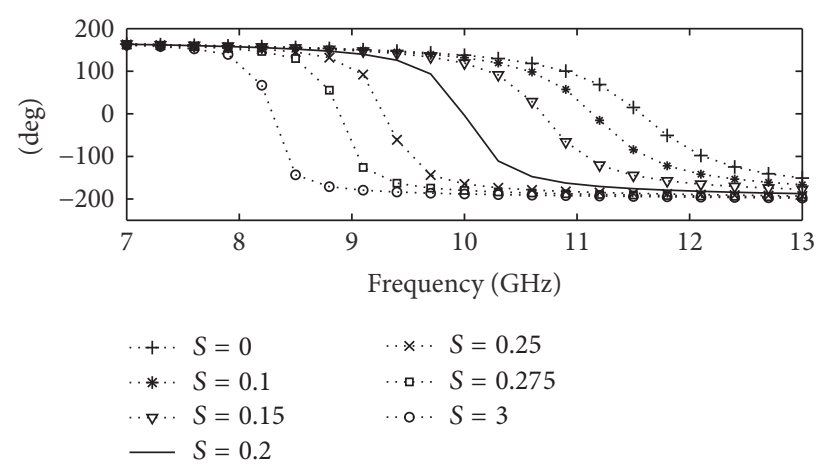

(a)

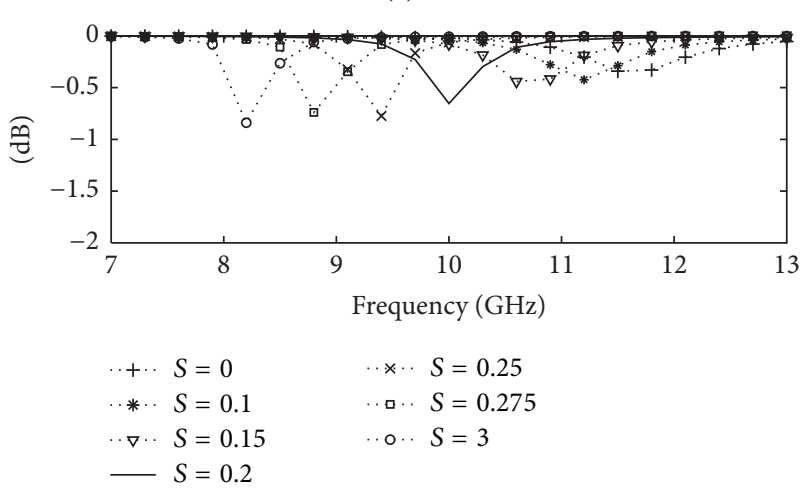

(b)

FIGURE 12: (a) Phase and (b) amplitude of the simulated reflection coefficient versus frequency for different value of the scaling factor $S$ (1st iteration fractal unit cell with $L=7.82 \mathrm{~mm}$ and $\Delta x \times \Delta y=$ $\left.0.4 \lambda_{0} \times 0.4 \lambda_{0}\right)$.

good miniaturization level, ranging from $14.7 \%$ to $17.53 \%$, is obtained for all designed fractal elements. The achieved miniaturization percentages approach the value of 32\% [19], if the fractals sizes are compared with the maximum square patch size, necessary to effectively tune the reflection phase of the $0.6 \lambda_{0}$ cell (i.e., $+20 \%$ patch resonant length).

Table 2 shows that, for fixed cell sizes, both the phase ranges as well as the maximum reflection losses increase, when a fractal patch is considered. However, in this latter case the losses can be effectively reduced by adopting a smaller cell

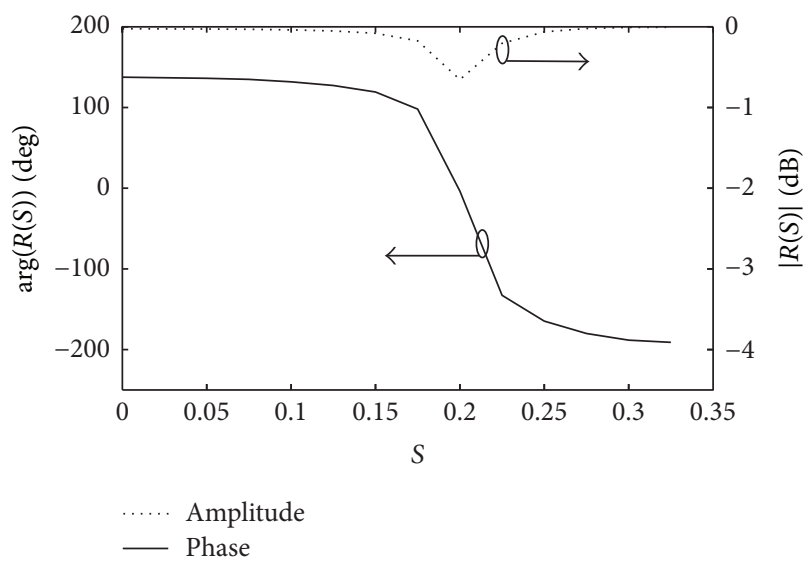

FIgURE 13: Simulated reflection coefficient versus $S(L=7.82 \mathrm{~mm}$, $\Delta x \times \Delta y=0.4 \lambda_{0} \times 0.4 \lambda_{0}$, and $f_{0}=10 \mathrm{GHz}$ ).

size, with a very low impact on the phase ranges, still remaining quite good. Furthermore, it is interesting to observe that, by reducing the unit cell size, a significant improvement is obtained in the reflectarray element bandwidth, which increases from $1.8 \%\left(0.5 \lambda_{0}\right.$ cell $)$ up to $3.6 \%\left(0.3 \lambda_{0}\right.$ cell $)$ [22-24]. As a matter of fact, the frequency variation of the reflection coefficients depicted in Figures 10, 12, and 14 show a slowly varying behavior for smaller values of the cell size. This last feature has an effect also on the $10 \mathrm{GHz}$ design phase curves $R(S)$, computed versus the scaling factor $S$ (Figures 11, 13, and 15). It can be observed that both the phase range and the curve slope are reduced, when the cell size varies from $0.5 \lambda$ down to $0.3 \lambda$. This behavior contributes to reducing phase errors due to the accuracy of the fabrication process that will have a minor impact on the reflectarray performances.

3.3. Design of 2 nd Iteration Fractal Cells. Starting from the fractal unit cells described in the previous paragraph, the corresponding 2nd iteration fractal elements are properly designed (Figure 6(c)). The antennas side length $L$, giving the resonance at $f_{0}=10 \mathrm{GHz}$ for $S=0.2$, is equal to $7.72 \mathrm{~mm}$, $7.68 \mathrm{~mm}$, and $7.51 \mathrm{~mm}$, respectively, for the considered unit cell sizes $0.5 \lambda_{0} \times 0.5 \lambda_{0}, 0.4 \lambda_{0} \times 0.4 \lambda_{0}$, and $0.3 \lambda_{0} \times 0.3 \lambda_{0}$. 


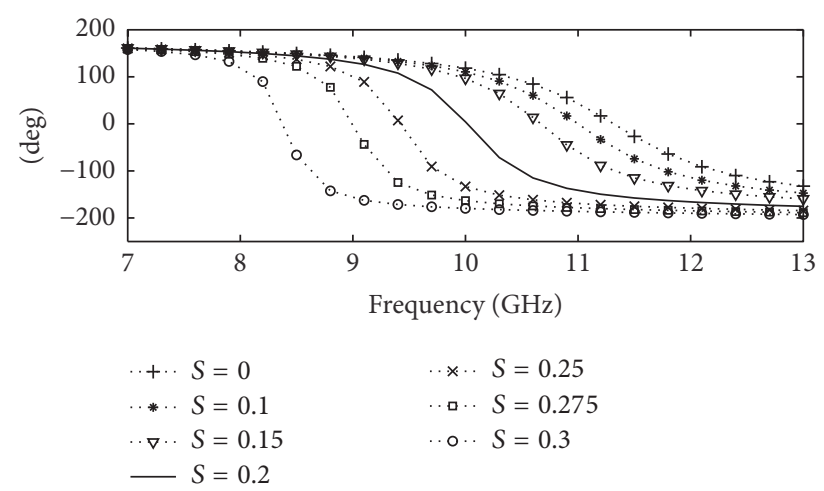

(a)

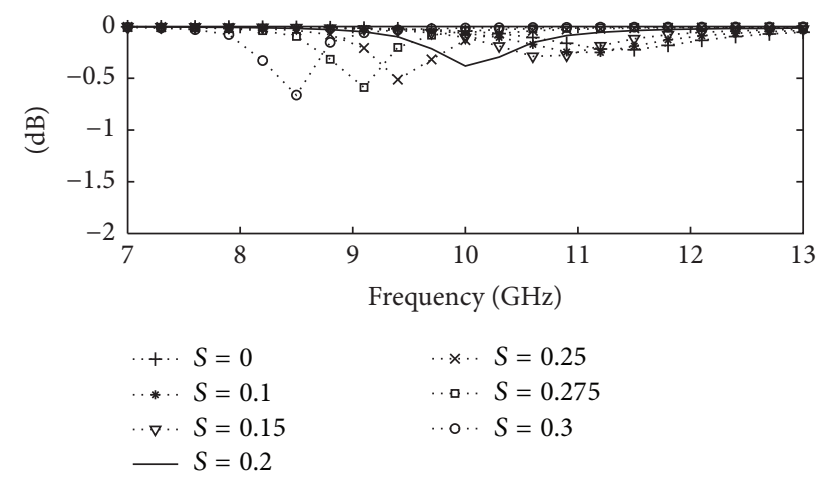

(b)

FIGURE 14: (a) Phase and (b) amplitude of the simulated reflection coefficient versus frequency for different value of the scaling factor $S$ (1st iteration fractal unit cell with $L=7.6 \mathrm{~mm}$ and $\Delta x \times \Delta y=$ $\left.0.3 \lambda_{0} \times 0.3 \lambda_{0}\right)$.

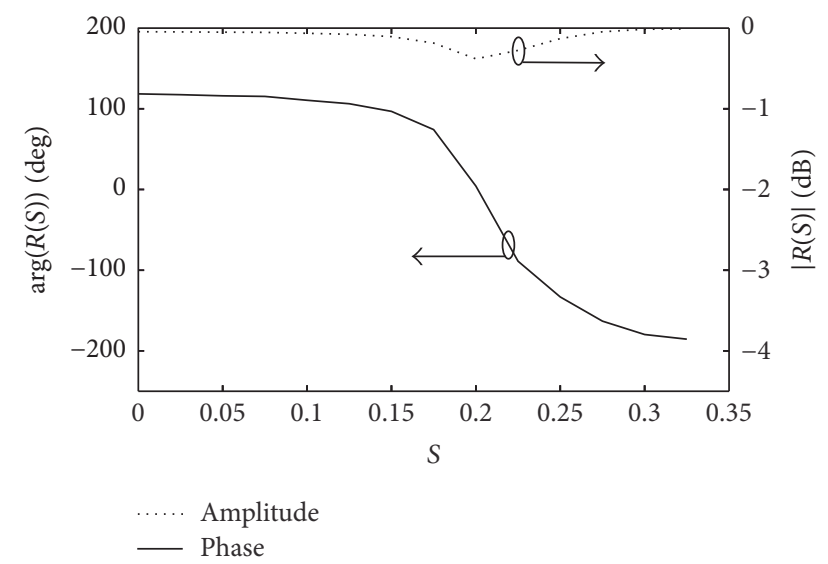

FIGURE 15: Simulated reflection coefficient versus $S(L=7.6 \mathrm{~mm}$, $\Delta x \times \Delta y=0.3 \lambda_{0} \times 0.3 \lambda_{0}$, and $f_{0}=10 \mathrm{GHz}$ ).

As in the previous cases, all patches are printed onto a Diclad 870 substrate with a dielectric constant $\varepsilon_{r}=2.33$ and a thickness $h=0.762 \mathrm{~mm}$ (Figure $6(\mathrm{~d})$ ). The design phase curves computed for each considered unit cell are reported under Figure 16. It can be observed that, by varying the scaling factor $S$ from 0 up to 0.325 , a good phase tuning range is obtained for each reflectarray cell. Table 3 summarizes

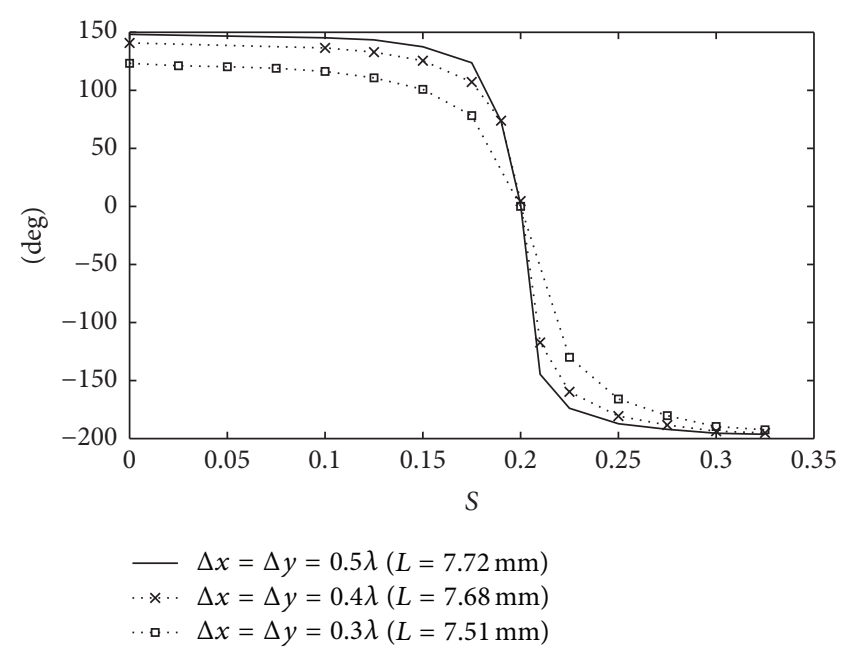

FIGURE 16: Comparison between simulated phase curve versus $S$ for different values of the unit cell size (2nd iteration).

TABLE 3: Fractal unit cell performances (2nd iteration Minkowski patch).

\begin{tabular}{lcccc}
\hline $\begin{array}{l}\text { Cell size } \\
\Delta x \times \Delta y\end{array}$ & $\begin{array}{c}\text { Patch } \\
\text { length } \\
L[\mathrm{~mm}]\end{array}$ & $\begin{array}{c}\text { Phase } \\
\text { range } \\
{[\mathrm{deg}]}\end{array}$ & $\begin{array}{c}\text { Max } \\
\text { loss } \\
{[\mathrm{dB}]}\end{array}$ & $\begin{array}{c}\text { Size reduction } \\
\text { (with respect to the } \\
0.6 \lambda \text { resonant square } \\
\text { patch in Table 2) }\end{array}$ \\
\hline $0.5 \lambda \times 0.5 \lambda$ & 7.72 & 348 & 1.12 & $16.22 \%$ \\
$0.4 \lambda \times 0.4 \lambda$ & 7.68 & 345 & 0.73 & $16.66 \%$ \\
$0.3 \lambda \times 0.3 \lambda$ & 7.51 & 330 & 0.42 & $18.50 \%$ \\
\hline
\end{tabular}

the features of the synthesized unit cells in terms of size reduction, phase range, and losses. It can be observed that the benefits due to the use of a 2nd iteration fractal configuration are very limited. As matter of fact, the phase tuning range and the percentage size reduction are little larger with respect to the corresponding 1st iteration fractal cell (see Table 2). Taking into account the higher complexity as well as the major fabrication precision related to 2 nd iteration fractal patch, in this work it seems convenient to stop the generation of the fractal unit cells at the first iteration. However, even the small improvements due to the 2 nd iteration patch could be fruitfully exploited for reflectarrays with a lower operating frequency. As a matter of fact, when the design frequency is smaller, the sizes of the fractal insets $\left(\sim S^{2} L\right)$ are greater, thus a minor precision level is required in the prototypes fabrication.

\section{Design and Measurements of Reflectarrays Based on Fixed-Size Fractal Radiators with Reduced Interelement Spacing}

To prove the effectiveness of the proposed fractal-shaped reflectarray configuration, various prototypes composed by $15 \times 15$ radiators are designed at $f_{0}=10 \mathrm{GHz}$.

In particular, the designed 1st iteration fractal cell, characterized by $\Delta x \times \Delta y=0.3 \lambda_{0} \times 0.3 \lambda_{0}$ (see Section 3.2 for details), 


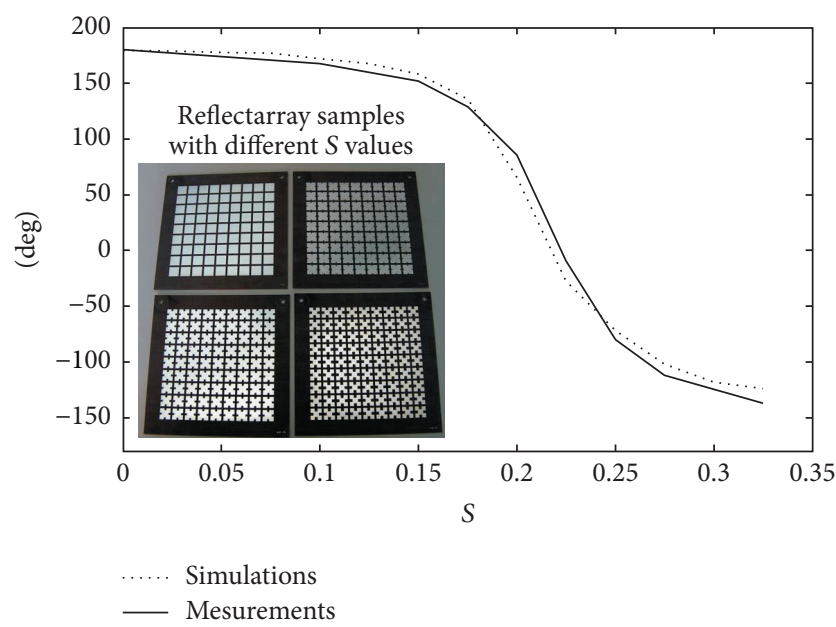

FIgURE 17: Comparison between simulated and measured phase curves versus $S\left(L=7.6 \mathrm{~mm}, \Delta x \times \Delta y=0.3 \lambda_{0} \times 0.3 \lambda_{0}\right.$, and $\left.f_{0}=10 \mathrm{GHz}\right)$.

is considered as elementary cell. As highlighted above, the adoption of a reduced cell size is finalized to the design of a reflectarray able to point one or multiple main beams along very wide angular directions off-broadside within a large frequency band. In this way, the miniaturization capabilities offered by the adopted fractal geometry are fruitfully exploited to improve the scanning capabilities, as well as to enlarge the antenna bandwidth. The following sections describe, respectively, the unit cell validation test, the synthesis algorithm adopted to design reflectarrays and finally the experimental validation on a reflectarray prototype.

4.1. Experimental Validation of the Unit Cell. In order to experimentally derive the reflectarray phase design curve, the miniaturized $0.3 \lambda_{0} \times 0.3 \lambda_{0}$ cell is tested with a measurement system based on the use of a transmitting and a receiving horn antenna, both connected to a vector network analyzer [19]. The arranged measurement setup allows us to detect the amplitude and phase of the far-field reflected by the antenna along broadside, when it is illuminated by a normally incident plane wave. The directivity of the test is properly increased in order to reduce the measurements sensitivity to the external noise. To this end, a set of small arrays (Figure 17), composed by $9 \times 9$ identical elements, are considered as reflectarray unit cell. Each array sample is characterized by a different scaling factor $S$. Figures 17 and 18 show the comparison between simulations and measurements. In particular, Figure 17 depicts the reflection phase curves versus the parameter $S$, which are simulated and measured at the working frequency $f_{0}=10 \mathrm{GHz}$, while Figure 18 illustrates the comparisons between the curves obtained for different values of $S$, within the frequency range starting from $9 \mathrm{GHz}$ up to $11 \mathrm{GHz}$. Both figures show a good agreement between simulated and measured data, thus demonstrating the effectiveness of the proposed miniaturized cell. Furthermore, a full phase range can be observed at the central frequency of

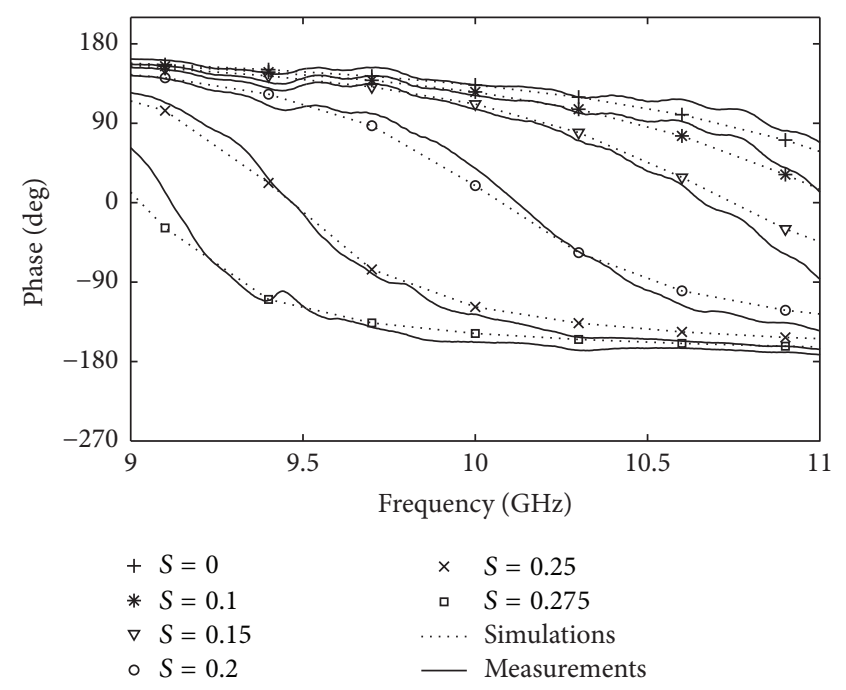

FIGURE 18: Comparison between simulated and measured phase curves versus frequency for values of $S(L=7.6 \mathrm{~mm}, \Delta x \times \Delta y=$ $\left.0.3 \lambda_{0} \times 0.3 \lambda_{0}\right)$.

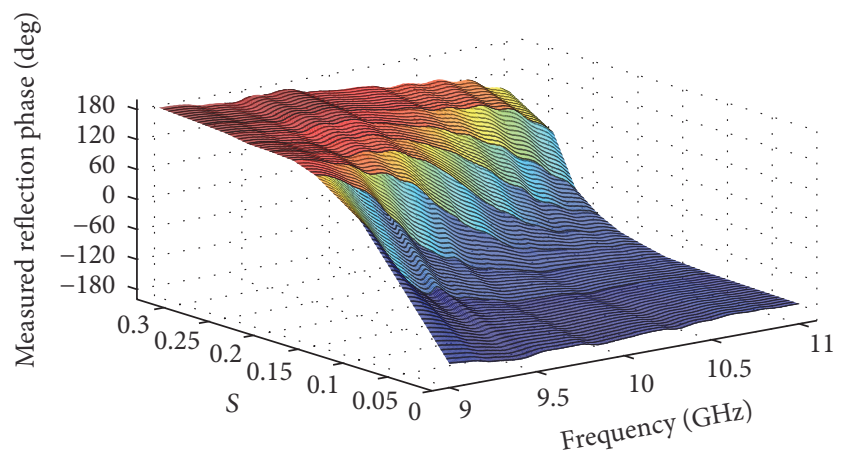

FIGURE 19: Measured phase curve versus frequency and scaling factor $S\left(L=7.6 \mathrm{~mm}, \Delta x \times \Delta y=0.3 \lambda_{0} \times 0.3 \lambda_{0}\right)$.

$10 \mathrm{GHz}$, while good phase tuning capabilities are obtained within the frequency interval [9-11] GHz (Figure 19).

4.2. Reflectarray Synthesis Algorithm. A general scheme for the designed reflectarrays is illustrated in Figure 20. As aforementioned, each antenna is composed by an array of 15 $\times 15$ fractal elements with an interelements spacing of $0.3 \lambda_{0} \times$ $0.3 \lambda_{0}$ at the frequency of $10 \mathrm{GHz}$. The array is illuminated by an X-band pyramidal horn, having an offset angle of $15^{\circ}$ with respect to the direction normal to the array surface, in the $E$-plane (i.e., $(y-z)$-plane in Figure 20). The horn, having an aperture size equal to $4.8 \mathrm{~mm} \times 5 \mathrm{~mm}$, is placed at a distance equal to $50 \mathrm{~cm}$ from the array.

As it can be observed in the front view of a reflecting surface example (Figure 20), each array element is characterized by a different fractal size, namely, a specific scaling factor $S_{\mathrm{nm}}$, which is properly chosen to compensate for the phase delay in the field coming from the feed and to obtain also a prescribed pattern. A synthesis algorithm based on the iterative projection method $[27,28]$ is applied to compute 


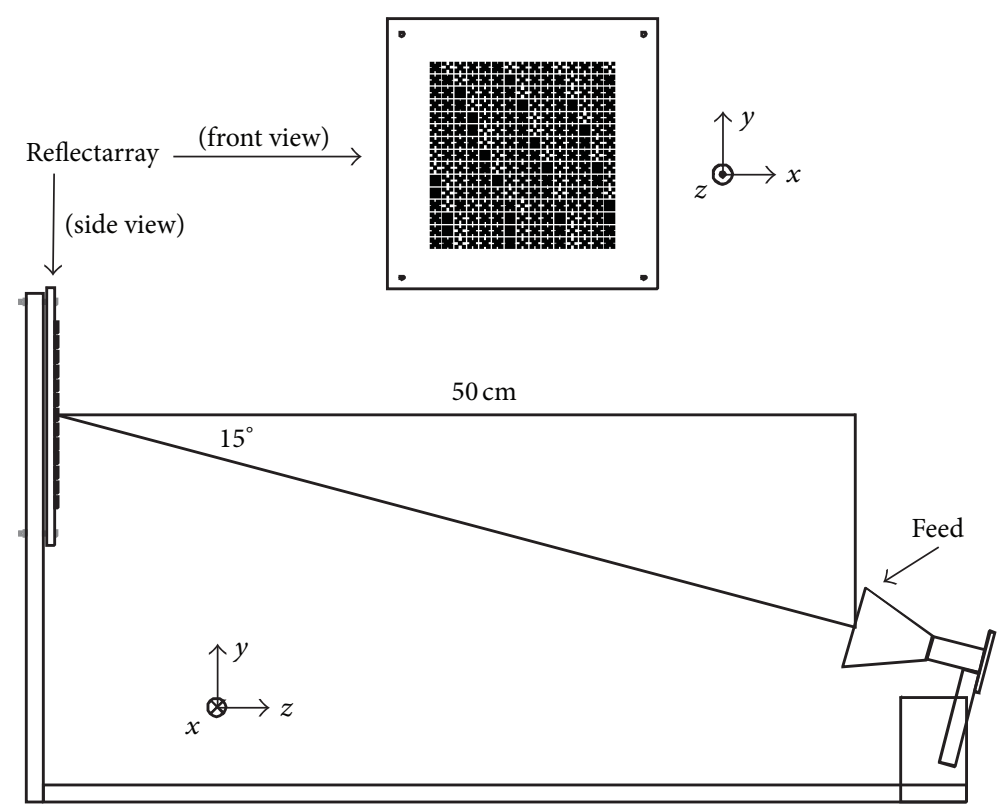

FIGURE 20: Reflectarrays layout.

the phase distribution on the array elements satisfying the imposed design goals.

The synthesis problem is formulated as the search of an array factor, namely, the array excitation coefficients $a_{\mathrm{nm}}$, giving the desired radiation pattern shape. It finds a feasible solution within the intersection $M \cap B$, where $B$ is the set containing the allowable excitation coefficients, related to the reflection behavior of the reflectarray unit cell, while $M$ is the set of array factors satisfying the pattern requirements, which are usually defined as the patterns lying between a lower and an upper bound masks.

The sets $B$ and $M$ adopted in this work are, respectively, the unit cell reflection coefficient $R(S)$ reported in Figure 15 and any patterns lying between a couple of masks that impose the desired pattern behavior as in the examples illustrated in the following (Figures 21(a)-24(a)). The algorithm output coefficients are finally adopted to select the scaling factors $S_{\mathrm{nm}}$, in order to draw the fractal reflectarray elements. To this end, a research routine is implemented to fit each $a_{\mathrm{nm}}$ coefficient onto the complex design curve $R(S)$, in order to extract the corresponding $S_{\mathrm{nm}}$ factor. In particular, searching for the phase of each $a_{\mathrm{nm}}$ coefficient on the $\arg (R(S))$-curve (Figure 15), the corresponding $S_{\mathrm{nm}}$ value is found through the projection onto the abscissa axis; namely,

$$
S_{\mathrm{nm}}=S_{i}: \arg \left(R\left(S_{i}\right)\right)=\arg \left(a_{\mathrm{nm}}\right), \quad \mathrm{S}_{i} \in[0-0.325] .
$$

The algorithm is adopted to design different reflectarrays characterized by the following features in the $H$-plane: a single main beam directed along $50^{\circ}$ off-broadside (design $\# 1$ ); two specular beams directed along $-30^{\circ}$ and 30 (design \#2); two main beams pointed towards $20^{\circ}$ and $50^{\circ}$ directions (design \#3); and finally a three-beam pattern which focuses the field along $-30^{\circ}, 0^{\circ}$, and $30^{\circ}$ directions (design \#4).

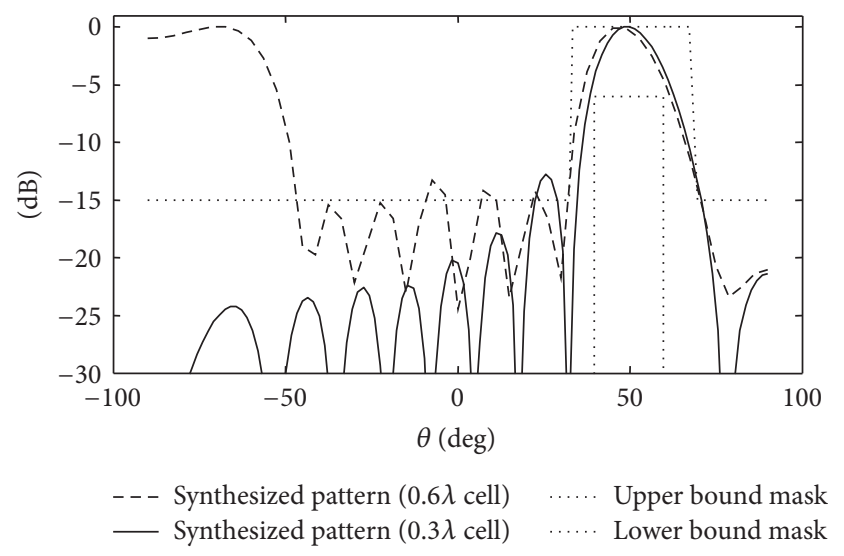

(a)

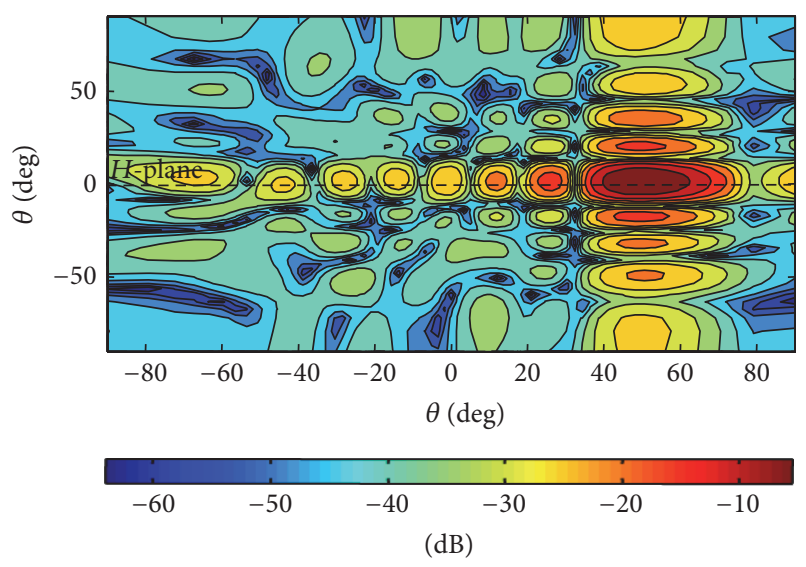

(b)

Figure 21: Design \#1: (a) synthesized $H$-plane array factor; (b) synthesized $E$-field contour plot. 


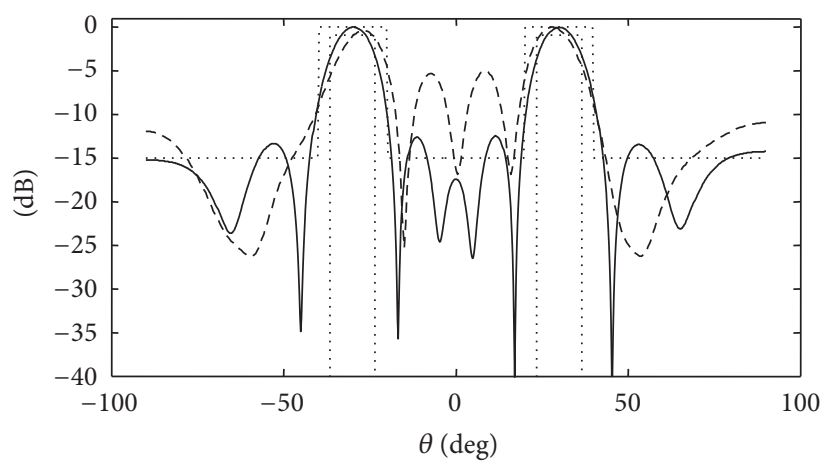

- - Synthesized pattern $(0.6 \lambda$ cell $) \quad \ldots .$. Upper bound mask — Synthesized pattern $(0.3 \lambda$ cell $) \quad \ldots .$. Lower bound mask

(a)

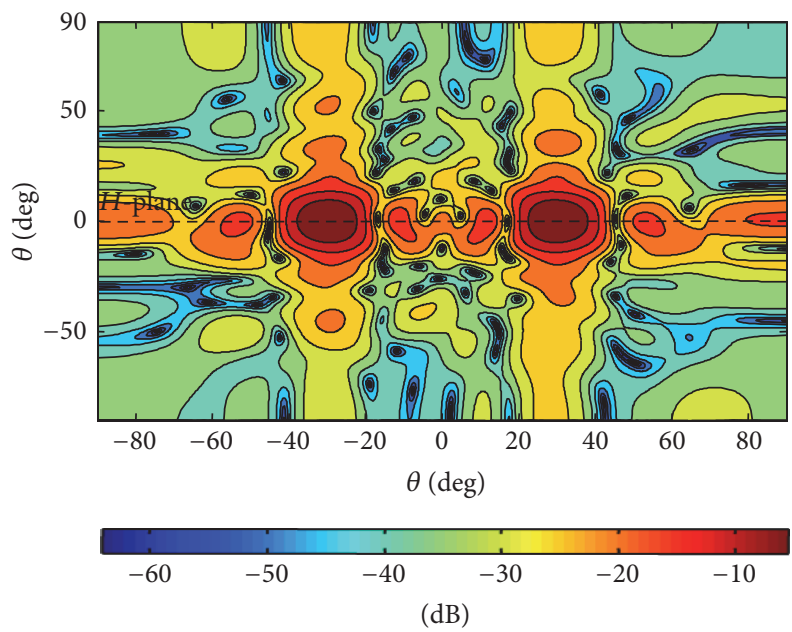

(b)

Figure 22: Design \#2: (a) synthesized $H$-plane array factor; (b) synthesized $E$-field contour plot.

By imposing the bound masks depicted, respectively, in Figures 21(a)-24(a) and adopting the design curve illustrated in Figure 15, the synthesis algorithm returns the $H$-plane array factors which are properly reported in same figures. Furthermore, the synthesized $E$-field contour plots computed for each design are illustrated in Figures 21(b)-24(b), respectively. A very good agreement is obtained between each synthesized pattern and the constraints imposed by the algorithm. Each pattern is compared also with the corresponding synthesized field relative to a $0.6 \lambda \times 0.6 \lambda$ fractal reflectarray having the same reflecting surface size (i.e., composed by $7 \times 7$ elements). In these latter cases, the presence of high side lobes or undesired grating lobes can be observed, which are effectively avoided through the use of the miniaturized fractal-shaped cell.

4.3. Reflectarray Realization and Test. The $0.3 \lambda \times 0.3 \lambda$ spaced reflectarray synthesized in design \#1 (Figure 21) is realized and tested to prove the effectiveness of the proposed configuration. In particular, the capability to point to the main

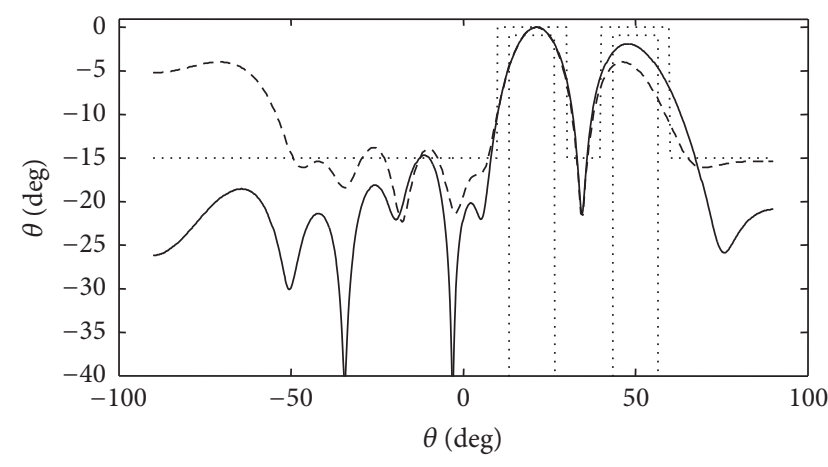

- - - Synthesized pattern $(0.6 \lambda$ cell $) \quad \ldots .$. Upper bound mask — Synthesized pattern $(0.3 \lambda$ cell $) \quad \ldots .$. Lower bound mask

(a)

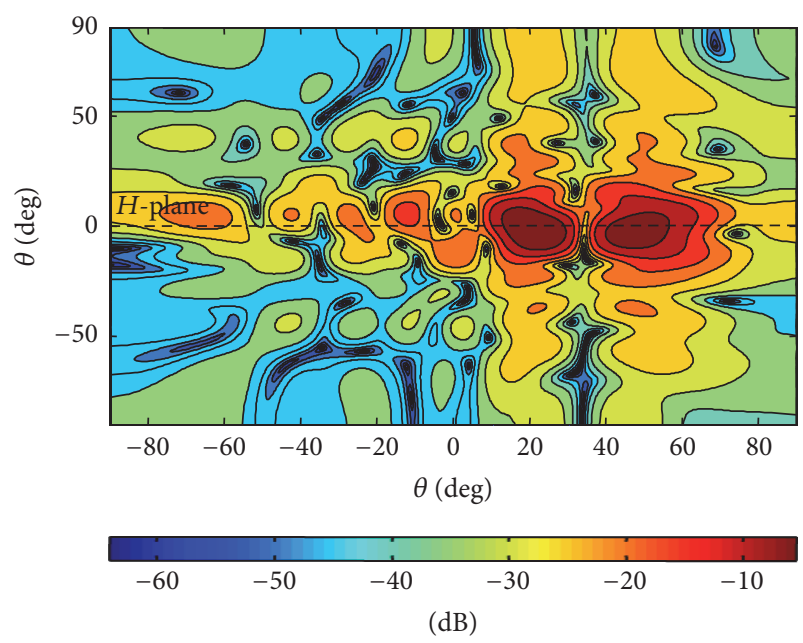

(b)

Figure 23: Design \#3: (a) synthesized $H$-plane array factor; (b) synthesized $E$-field contour plot.

lobe along a very far angular direction within a quite large frequency band is experimentally verified.

Prior to realize the antenna, the bandwidth performances are numerically checked by computing the normalized gain pattern versus frequency along the maximum radiation direction (i.e., $50^{\circ}$ ). The pattern reported in Figure 25 shows a quite good $-1 \mathrm{~dB}$ gain of about $6 \%$. In order to evaluate the bandwidth improvement due to the use of a smaller cell size, in the same figure the gain patterns computed for two differently spaced fractal-reflectarrays are reported.

The above antennas are, respectively, composed by $11 \times$ 11 fractal elements embedded into a $0.4 \lambda$ cell and $9 \times 9$ radiators embedded into a greater $0.5 \lambda$ cell, thus having the same aperture size (i.e., $\cong 4.5 \lambda \times 4.5 \lambda$ ) of the designed $0.3 \lambda$ reflectarray prototype.

Both antennas are synthesized in order to always point to the main beam along $50^{\circ}$ in the $H$-plane, by adopting the phase design curves reported in Figures 13 and 11, respectively. The gain patterns depicted in Figure 25 show an increasing $-1 \mathrm{~dB}$ gain bandwidth when the unit cell size is progressively reduced. In particular, the bandwidth is equal 


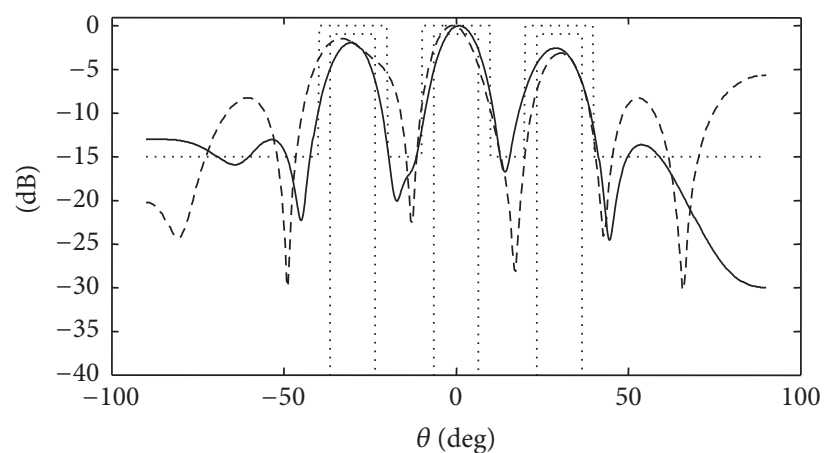

- - - Synthesized pattern $(0.6 \lambda$ cell $) \quad \ldots$. Upper bound mask — Synthesized pattern $(0.3 \lambda$ cell $) \quad \ldots .$. Lower bound mask

(a)

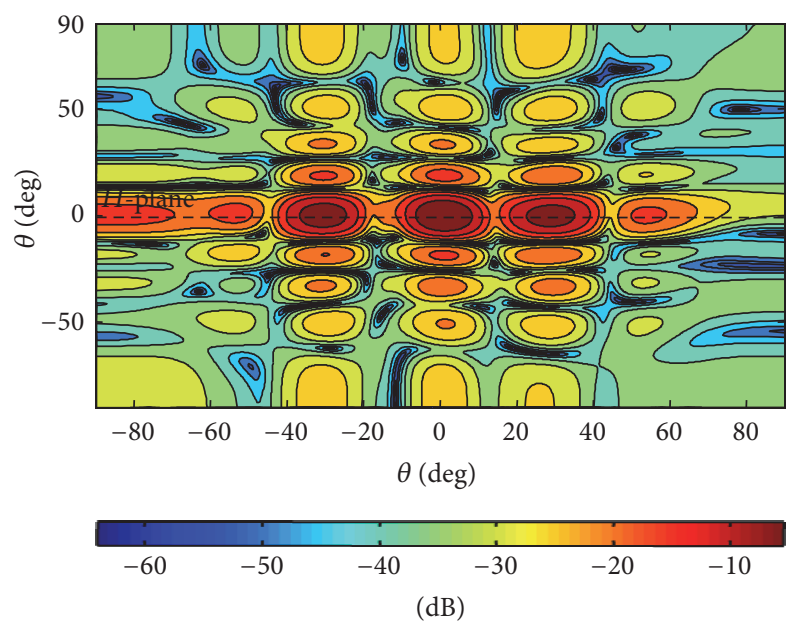

(b)

Figure 24: Design \#4: (a) synthesized $H$-plane array factor; (b) synthesized $E$-field contour plot.

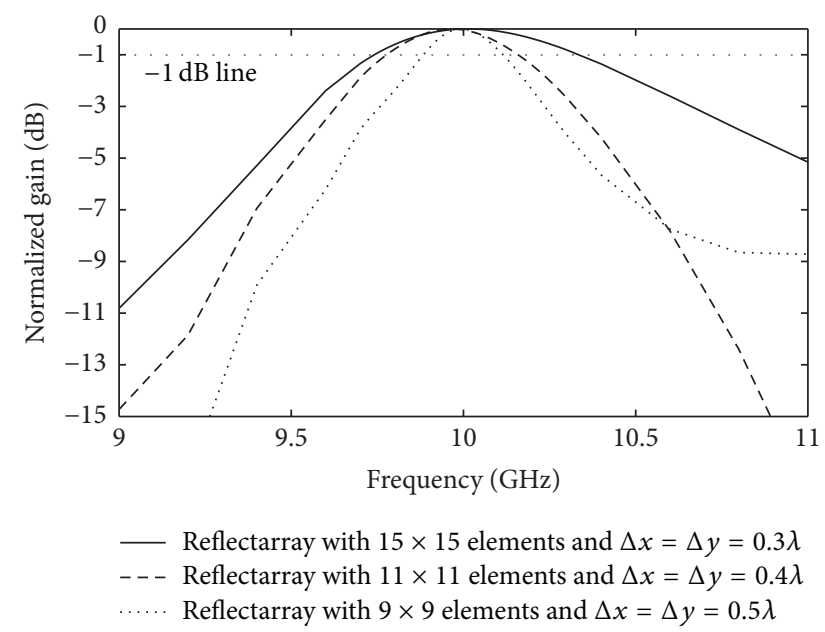

FIGURE 25: Comparison between computed gain patterns versus frequency of differently spaced reflectarrays.

to $2.3 \%$ for $\Delta x=\Delta y=0.5 \lambda, 3.8 \%$ for $\Delta x=\Delta y=0.4 \lambda$, and $6 \%$ for $\Delta x=\Delta y=0.3 \lambda$, thus achieving an improvement

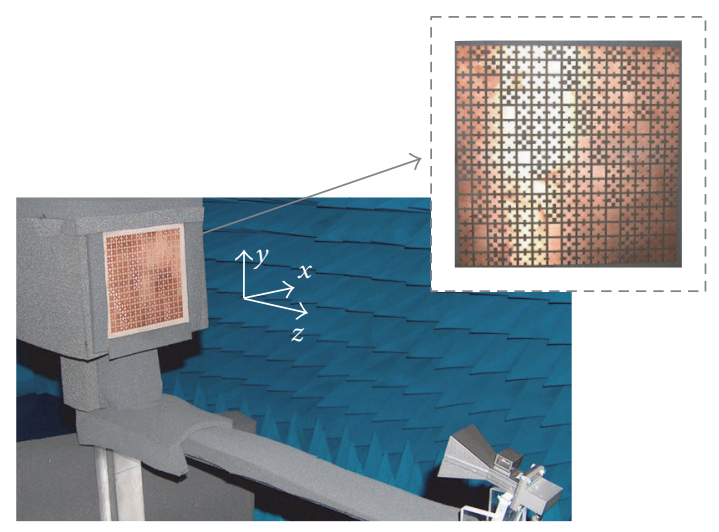

FIGURE 26: Photograph of the fabricated reflectarray prototype.

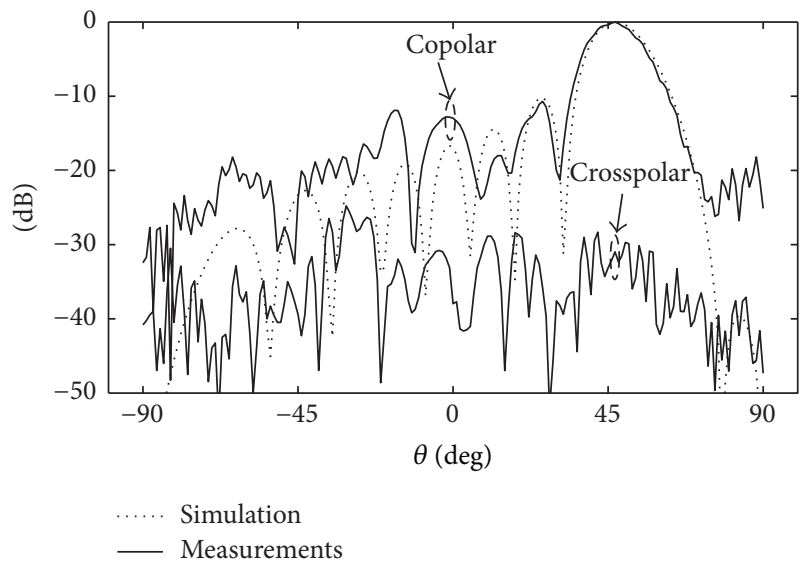

FIGURE 27: Comparison between measured and simulated radiation patterns.

of about 2.5 times with respect to the standard half-spaced reflectarrays.

Finally, the scaling factors $S_{\mathrm{nm}}$, computed by the $0.3 \lambda \times$ $0.3 \lambda$ reflectarray synthesis, are adopted to draw the array elements giving the desired behavior, thus obtaining the fabricated prototype illustrated in Figure 26.

The realized reflectarray prototype is tested into the anechoic chamber of the University of Calabria, with a standard X-band horn adopted as measurement probe. The reflectarray antenna is placed in its far-field region at a distance of about $2.5 \mathrm{~m}$ from the probe aperture.

The radiation pattern, measured at the frequency of $10 \mathrm{GHz}$ (Figure 27), shows a good agreement with the constraints imposed to the main beam position during the synthesis step. In particular, the measured pattern very well agrees with the simulated one, showing the effectiveness of the proposed miniaturized fractal-shaped cell. Furthermore, it can be observed that the measured cross-polar components have a lower intensity level (about $25 \mathrm{~dB}$ ) with respect to the copolar pattern main lobe.

The gain pattern versus frequency measured along the maximum radiation direction (i.e., $\cong 50^{\circ}$ ) is reported in Figure 28. A little frequency shift is observed with respect to the simulated pattern (Figure 25) while similar bandwidth 


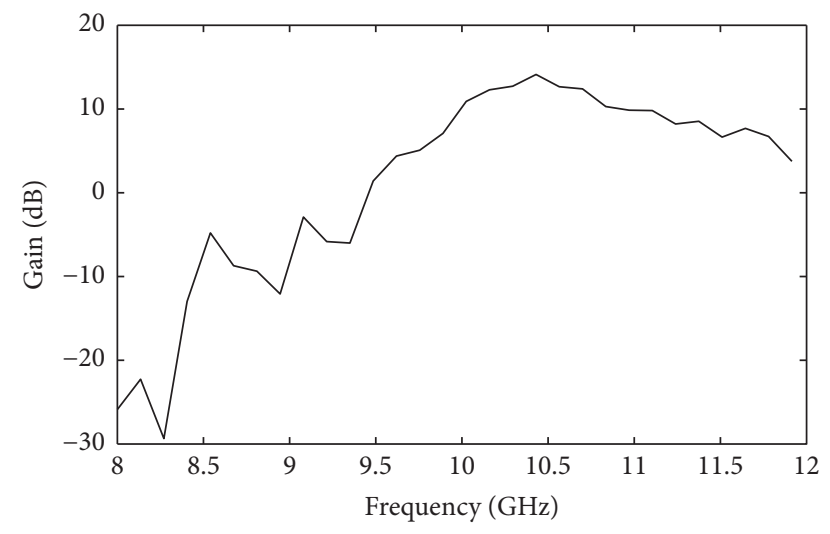

FIGURE 28: Measured gain pattern versus frequency.

performances can be appreciated. The low measured gain is due to presence of very high spillover losses, caused by the small size of the reflecting surface. However, the aim of the implemented experimental test is to prove the effectiveness of the proposed miniaturized fractal cell in the design of reflectarray antennas characterized by wide main-beam positions, within a moderate large frequency band. Consequently, the other performances of the realized reflectarray prototype are not adequately optimized.

\section{Conclusions}

A careful review of fractal-shaped reflectarray configurations has been presented in this paper, by identifying both benefits as well as limitations.

Furthermore, an extensive analysis of a Minkowski patch, offering new size reduction opportunities, has been presented.

The miniaturization capabilities of a fixed-size fractal patch configuration have been fully exploited to design compact-size microstrip reflectarrays offering quite large (up to 50 degrees) beam-pointing capabilities over a satisfactory frequency band (about 6\%). The design and experimental validations of a $10 \mathrm{GHz} 0.3 \lambda \times 0.3 \lambda$ unit cell have been fully described to prove the miniaturization effect, while preserving quite good phase agility (between 320 and 330 degrees). Starting from the basic fractal-shaped unit cell, a specific synthesis algorithm based on the iterative projection method has been applied to design various reflectarrays prototypes, offering single or multiple-beams capabilities over a large angular region. Fully experimental validations in terms of radiation pattern and antenna gain have been finally discussed on a realized fractal reflectarray prototype.

\section{Competing Interests}

The authors declare that there is no conflict of interests regarding the publication of this paper.

\section{References}

[1] J. Huang and J. Encinar, Reflectarray Antennas, Wiley-IEEE Press, New York, NY, USA, 2008.
[2] D. M. Pozar and T. A. Metzler, "Analysis of a reflectarray antenna using microstrip patches of variable size," Electronics Letters, vol. 29, no. 8, pp. 657-658, 1993.

[3] J. Huang, "Microstrip reflectarray," in Proceedings of the IEEE Antennas and Propagation Society International Symposium, pp. 612-615, Ontario, Canada, June 1991.

[4] J. Huang and R. J. Pogorzelski, "A ka-band microstrip reflectarray with elements having variable rotation angles," IEEE Transactions on Antennas and Propagation, vol. 46, no. 5, pp. 650-656, 1998.

[5] S. V. Hum, M. Okoniewski, and R. J. Davies, "Realizing an electronically tunable reflectarray using varactor diode-tuned elements," IEEE Microwave and Wireless Components Letters, vol. 15, no. 6, pp. 422-424, 2005.

[6] M. Riel and J.-J. Laurin, "Design of an electronically beam scanning reflectarray using aperture-coupled elements," IEEE Transactions on Antennas and Propagation, vol. 55, no. 5, pp. 1260-1266, 2007.

[7] F. Venneri, S. Costanzo, G. Di Massa, A. Borgia, P. Corsonello, and M. Salzano, "Design of a reconfigurable reflectarray based on a varactor tuned element," in Proceedings of the 6th European Conference on Antennas and Propagation (EuCAP '12), pp. 2628-2631, March 2012.

[8] F. Venneri, S. Costanzo, G. Di Massa et al., "Beam-scanning reflectarray based on a single varactor-tuned element," International Journal of Antennas and Propagation, vol. 2012, Article ID 290285, 5 pages, 2012.

[9] F. Venneri, S. Costanzo, and G. Di Massa, "Reconfigurable aperture-coupled reflectarray element tuned by single varactor diode," Electronics Letters, vol. 48, no. 2, pp. 68-69, 2012.

[10] F. Venneri, S. Costanzo, and G. Di Massa, "Design and validation of a reconfigurable single varactor-tuned reflectarray," IEEE Transactions on Antennas and Propagation, vol. 61, no. 2, pp. 635-645, 2013.

[11] B. Pinte, H. Legay, E. Girard, R. Gillard, M. Charrier, and A. Ziaei, "A reflectarray antenna in Ka-band with MEMS control," in Proceedings of the IEEE International Symposium on Phased Array Systems and Technology, Columbus, Ohio, USA, 2004.

[12] S. V. Hum, G. McFeetors, and M. Okoniewski, "Integrated MEMS reflectarray elements," in Proceedings of the 1st European Conference on Antennas and Propagation (EuCAP '06), pp. 1-6, Nice, France, November 2006.

[13] F. Zubir and M. K. A. Rahim, "Simulated fractals shape for unit cell reflectarray," in Proceedings of the Asia Pacific Microwave Conference (APMC '09), pp. 583-586, Singapore, December 2009.

[14] F. Zubir, M. K. A. Rahim, O. Ayop, A. Wahid, and H. A. Majid, "Design and analysis of microstrip reflectarray antenna with minkowski shape radiating element," Progress in Electromagnetics Research B, vol. 24, pp. 317-331, 2010.

[15] A. Wahid, M. K. A. Rahim, F. Zubir, and M. F. Ismail, "Dual layer microstrip reflectarray composed of two stacked arrays with minkowski and square shaped radiating element," Journal Teknologi (Sciences \& Engineering), vol. 58, pp. 13-18, 2012.

[16] K. H. Sayidmarie and M. E. Bialkowski, "Fractal unit cells of increased phasing range and low slopes for single-layer microstrip reflectarrays," IET Microwaves, Antennas \& Propagation, vol. 5, no. 11, pp. 1371-1379, 2011.

[17] D. Oloumi, S. Ebadi, A. Kordzadeh, A. Semnani, P. Mousavi, and X. Gong, "Miniaturized reflectarray unit cell using fractalshaped patch-slot configuration," IEEE Antennas and Wireless Propagation Letters, vol. 11, pp. 10-13, 2012. 
[18] S. Costanzo and F. Venneri, "Fractal shaped reflectarray element for wide angle scanning capabilities," in Proceedings of the IEEE Antennas and Propagation Society International Symposium (APSURSI '13), pp. 1554-1555, Orlando, Fla, USA, July 2013.

[19] S. Costanzo and F. Venneri, "Miniaturized fractal reflectarray element using fixed-size patch," IEEE Antennas and Wireless Propagation Letters, vol. 13, pp. 1437-1440, 2014.

[20] J. P. Gianvittorio and Y. Rahmat-Samii, "Fractal antennas: a novel antenna miniaturization technique, and applications," IEEE Antennas and Propagation Magazine, vol. 44, no. 1, pp. 2036, 2002.

[21] D. H. Werner and S. Ganguly, "An overview of fractal antenna engineering research," IEEE Antennas and Propagation Magazine, vol. 45, no. 1, pp. 38-57, 2003.

[22] S. Costanzo, F. Venneri, and G. Di Massa, "Bandwidth enhancement of aperture-coupled reflectarrays," Electronics Letters, vol. 42, no. 23, pp. 1320-1322, 2006.

[23] F. Venneri, S. Costanzo, G. Di Massa, and G. Amendola, "Aperture-coupled reflectarrays with enhanced bandwidth features," Journal of Electromagnetic Waves and Applications, vol. 22, no. 11-12, pp. 1527-1537, 2008.

[24] F. Venneri, S. Costanzo, and G. Di Massa, "Bandwidth behavior of closely spaced aperture-coupled reflectarrays," International Journal of Antennas and Propagation, vol. 2012, Article ID 846017, 11 pages, 2012.

[25] J. Romeu and Y. Rahmat-Samii, "Fractal FSS: a novel dual-band frequency selective surface," IEEE Transactions on Antennas and Propagation, vol. 48, no. 7, pp. 1097-1105, 2000.

[26] J. P. Gianvittorio, J. Romeu, S. Blanch, and Y. Rahmat-Samii, "Self-similar prefractal frequency selective surfaces for multiband and dual-polarized applications," IEEE Transactions on Antennas and Propagation, vol. 51, no. 11, pp. 3088-3096, 2003.

[27] S. Costanzo, F. Venneri, G. Di Massa, and G. Angiulli, “Synthesis of microstrip reflectarrays as planar scatterers for SAR interferometry," Electronics Letters, vol. 39, no. 3, pp. 266-267, 2003.

[28] F. Venneri, S. Costanzo, G. Di Massa, and G. Angiulli, "An improved synthesis algorithm for reflectarrays design," IEEE Antennas and Wireless Propagation Letters, vol. 4, no. 1, pp. 258261, 2005. 


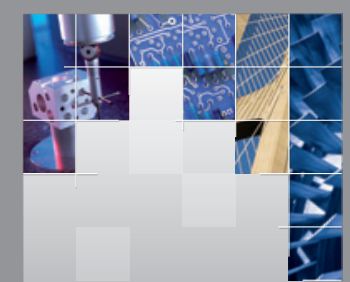

\section{Enfincering}
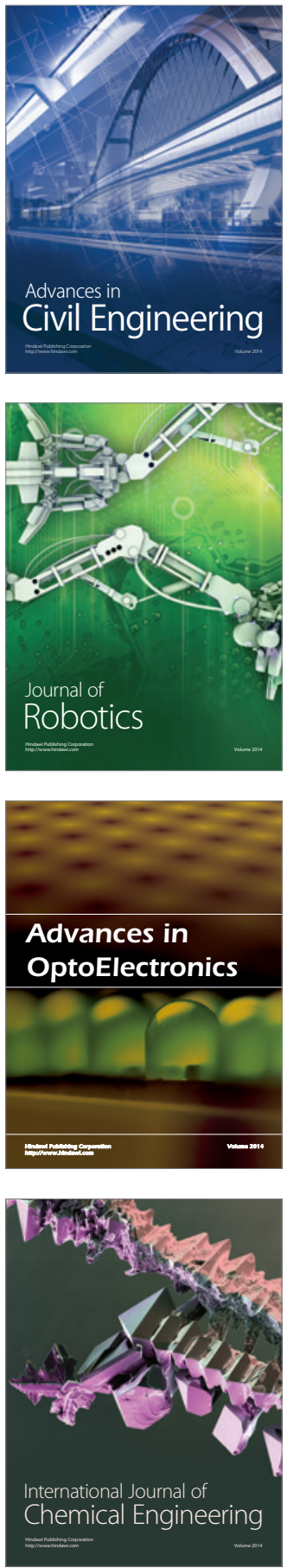

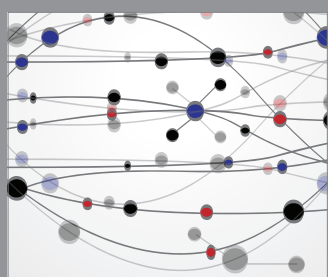

The Scientific World Journal

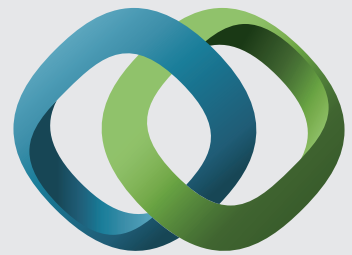

\section{Hindawi}

Submit your manuscripts at

http://www.hindawi.com
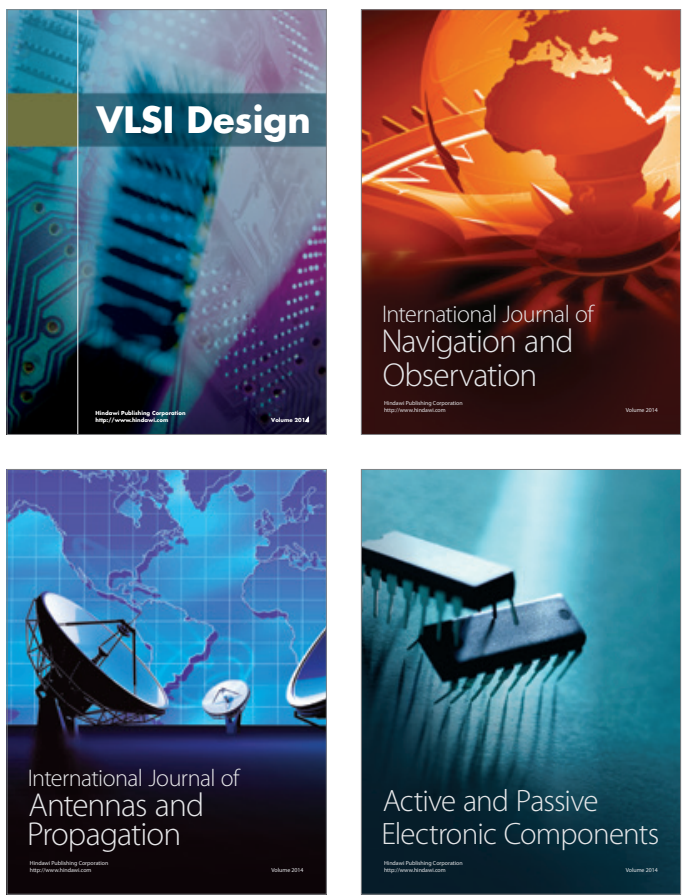
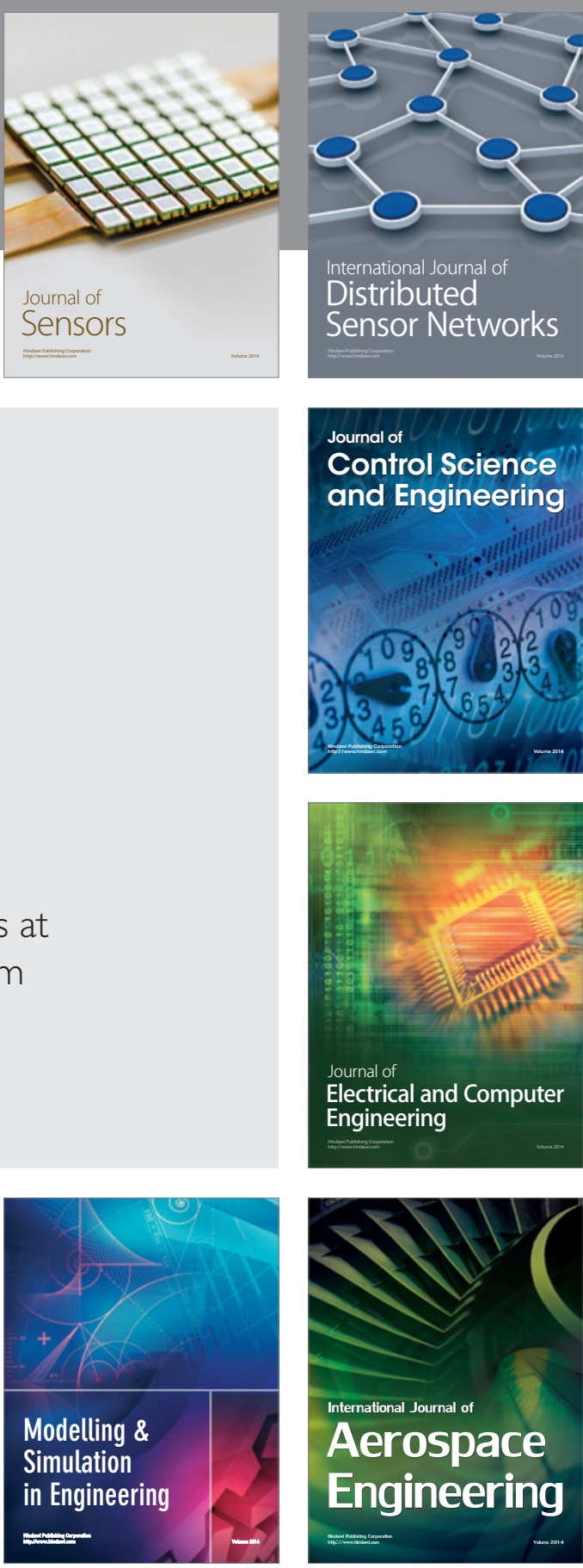

International Journal of

Distributed

Sensor Networks

Journal of

Control Science

and Engineering
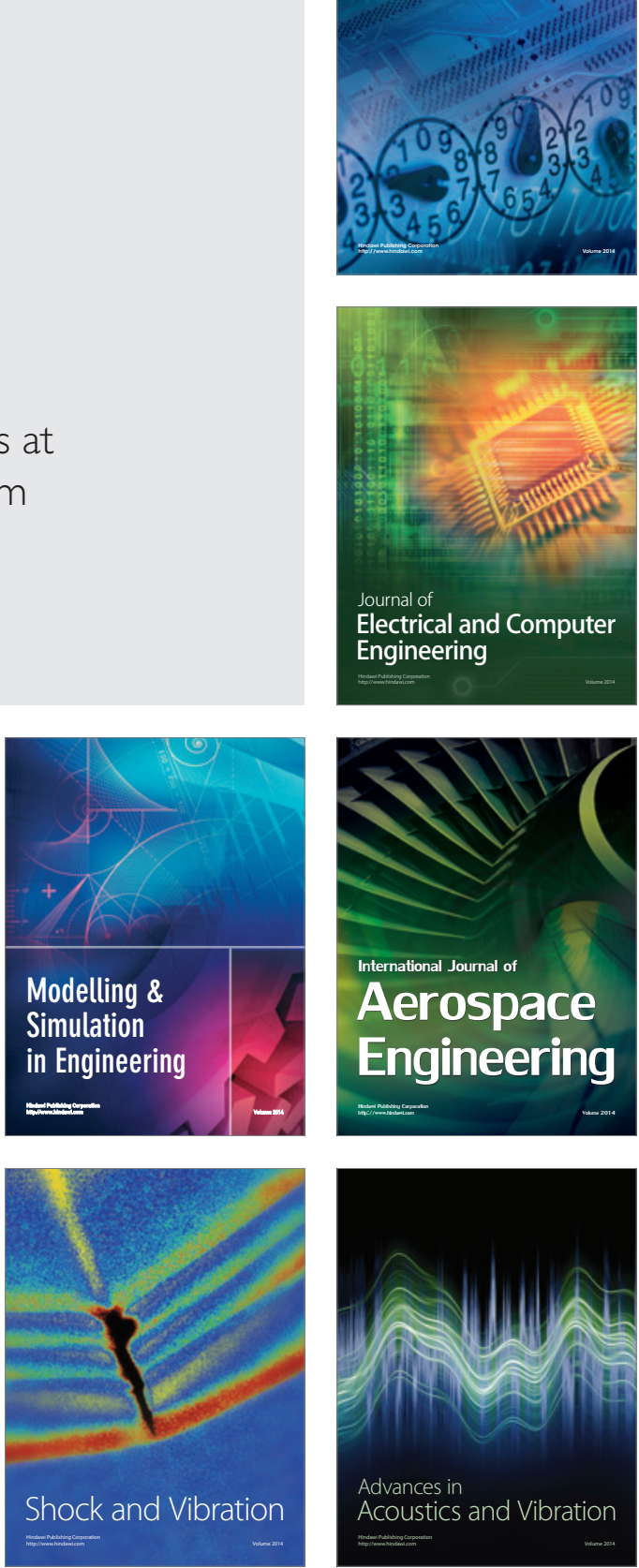